\title{
A Behavioral and Genetic Dissection of Two Forms of Olfactory Plasticity in Caenorhabditis elegans: Adaptation and Habituation
}

\author{
Nirit Bernhard and Derek van der Kooy ${ }^{1}$ \\ Neurobiology Research Group, Department of Anatomy and Cell Biology, University of Toronto, Ontario M5S-1A8, Canada
}

\begin{abstract}
Continuous presentation of an olfactory stimulus causes a decrement of the chemotaxis response in the nematode Caenorbabditis elegans. However, the differences between the learning process of habituation (a readily reversible decrease in behavioral response) and other types of olfactory plasticity such as adaptation (a decrement in response due to sensory fatigue, which cannot be dishabituated) have not been addressed. The volatile odorant diacetyl (DA) was used within a single paradigm to assess the distinct processes of olfactory adaptation and habituation. Preexposing and testing worms to $100 \%$ DA vapors caused a chemotaxis decrement that was not reversible despite the presentation of potentially dishabituating stimuli. This DA adaptation was abolished in worms with an odr-10 mutation (encoding a high-affinity DA receptor on the AWA neuron), even though naive chemotaxis remained unaffected. Conversely, DA adaptation remained intact in odr-1 mutants (defective in AWC neuron-mediated olfactory behavior), even though naive chemotaxis to DA decreased. Surprisingly, exposure to vapors of intermediate concentrations of DA $(0.01 \%$ and 25\%) did not cause worms to exhibit any response decrement. In contrast to preexposure to high DA concentrations, preexposure to low DA concentrations $(0.001 \%)$ produced habituation of the chemotaxis response (a dishabituating stimulus could reverse the response decrement back to baseline levels). The distinct behavioral effects produced by DA preexposure highlight a concentration-dependent dissociation between two decremental olfactory processes: adaptation at high DA concentrations versus habituation at low DA concentrations.
\end{abstract}

Our understanding of the mechanisms underlying some forms of learning and memory have been greatly facilitated by the use of invertebrate model systems. Extensive studies on associative and nonassociative learning have been carried out in molluscs, such as Aplysia californica (Castellucci et al. 1970; Walter et al. 1979,1981; Carew and Sahley 1986) and Hermissenda crassicorniss (Rogers and Matzel 1995), as well as in other invertebrate species such as Drosophila melanogaster (Quinn et al. 1974; Tully and Quinn 1985; Corfas and Dudai 1988) and Apis mellifera carnica (Braun and Bicker 1992; Hammer 1997; Hellerstern et al. 1998). In addition to these, the nematode Caenorbabditis elegans has also been identified as a useful organism for the study of learning and memory (Hedgecock and Russell 1975; Rankin et al. 1990; Wen et al. 1997; Morrison et al. 1999). C. elegans has a relatively simple nervous system, composed of 302 neurons whose synapses have been mapped out fully by electron microscope serial sections (White et al. 1986; Hall and Russel 1991). Combined with its detailed physical map and a genome that is nearly fully sequenced (Sulston et al. 1992; C. elegans Sequencing Con-

${ }^{1}$ Corresponding author.

E-MAIL derek.van.der.kooy@utoronto.ca; FAX 416-978-3844. sortium 1998), C. elegans is well suited for identifying specific molecular pathways and genes that play a role in learning, memory, and other forms of behavioral plasticity.

The search for molecular and genetic components of learning and memory in any organism must first begin with a clear definition of the different types of learning being investigated and then distinguishing between learning and other forms of plasticity. Different temporal relationships between stimuli during training allows for the classification of two elementary types of learning: nonassociative and associative learning (Byrne 1987). Whereas associative learning involves a change in behavior due to specific temporal contingencies between stimuli or between a stimulus and a behavioral response, nonassociative learning involves modification of a behavior due to the presentation of a single cue (Carew and Sahley 1986; Brown 1998;). Types of nonassociative learning include sensitization, habituation, and dishabituation. Sensitization refers to the enhancement of a behavioral response caused by a strong and often noxious stimulus (Groves and Thompson 1970). Habituation is the decrement in response (independent of sensory/motor fatigue or adaptation) due to repeated or continuous presentations of a single stimulus (Harris 1943; Thompson and Spencer 1966; Groves and Thompson 1970). Conversely,

LEARNING \& MEMORY 7:199-212 @ 2000 by Cold Spring Harbor Laboratory Press ISSN1072-0502/00 \$5.00

$$
\begin{array}{lllllllllllllll}
L & E & A & R & N & I & N & G & \& & M & E & M & O & R & Y \\
\text { www.learnmem.org } & & &
\end{array}
$$


dishabituation is the return from the habituated response to baseline response levels following the presentation of a novel or noxious stimulus. Dishabituation may be an instance of sensitization superimposed on the habituated response (Groves and Thompson 1970) or may involve different processes from sensitization (Marcus et al. 1988; Cohen et al. 1997). The reestablishment of baseline response levels following a dishabituating stimulus is distinct from a spontaneous recovery of the habituated response, which occurs without a dishabituating stimulus after a prolonged period of time (Thompson and Spencer 1966).

Harris (1943) and Thompson and Spencer (1966) suggest that continuous or repeated stimulus presentation leading to sensory adaptation can be caused by decreased receptor activity (receptor adaptation) or limitations of effector response (effector fatigue). Response decrements that can be accounted for by either of these mechanisms are not referred to as habituation, as they indicate an inability to respond to the stimulus as opposed to an active learningassociated modulation in behavior. There are examples of neuroadaptation within the central nervous system (Ochoa et al. 1990), as well as in sensory systems such as the mechanosensory (Corfas and Dudai 1990), visual (Dizhoor et al. 1991; Kawamura and Murakami 1991), auditory (Rauschecker and Korte 1993), and olfactory systems (Kramer and Siegelbaum 1992; Dawson et al. 1993; Chen and Yau 1994; Kurahashi and Menini 1997; Leinders-Zufall et al. 1998). Whereas the olfactory studies focus mainly on the cellular changes that lead to modulations in sensory transduction, they do not assess the behavioral processes that lead to olfactory behavioral plasticity.

The ability of $C$. elegans to sense, approach, and discriminate between volatile odorants has been shown to be manipulatible both at the cellular and genetic levels (Bargmann et al. 1990,1993; Colbert and Bargmann 1997). Laser ablation studies have identified the AWA and AWC primary chemosensory neurons as responsible for mediating olfaction to specific attractive odorants, while forward mutagenesis of wild-type worms has identified novel genes involved in olfaction (Bargmann et al. 1993; Colbert and Bargmann 1995; Sengupta et al. 1996). The AWA neuron has been shown to mediate approach to pyrazine as well as lower concentrations of diacetyl (Bargmann et al. 1993), and the odr-10 gene, which encodes a seven transmembrane domain diacetyl receptor, is expressed in AWA (Sengupta et al. 1996; Troemel et al. 1997; Zhang et al. 1997). In addition, $o d r-3$ (a $G \alpha$-protein subunit proposed to be involved in downstream intracellular signaling) and osm-9 (a putative ion channel with some homology to the Drosopbila transient receptor potential protein) have been identified and characterized behaviorally as affecting AWA olfactory function (Colbert et al. 1997; Roayaie et al. 1998). Laser and genetic ablations of the AWC neuron indicate that this neuron mediates responses to odorants such as benzaldehyde
(BZ), butanone, and isoamyl alcohol (Bargmann et al. 1993). In addition to the high-affinity $o d r$-10 diacetyl receptor on AWA, there appears to be a putative low-affinity diacetyl receptor on AWC that mediates responses to high concentrations (100\%) of DA and 2,3-pentanedione (Chou et al. 1996). Approach to high concentrations of DA remains intact after genetic ablation of all neurons except for AWC (Chou et al. 1996). Laser ablation of the AWC neuron results in a $15 \%$ decrease in approach to intermediate DA concentrations $(0.1 \% \mathrm{DA})$ that does not reach statistical significance, and laser ablation of AWA and AWC together does not completely eliminate approach toward $0.1 \%$ DA, suggesting that perhaps some other, unknown chemosensory neuron(s) may be involved in the residual response to this stimulus (Bargmann et al. 1993). In addition, odr-1 mutants, which demonstrate deficits in chemotaxis to AWC-sensed odorants also show lower than wild-type approach to intermediate DA concentrations (10\% DA and 1\% DA) (Bargmann et al. 1993). The putative low-affinity DA receptor on AWC may signal to a downstream G-protein coupled receptor through $o d r$-1 and lead to the opening of a cyclic nucleotide-gated cation channel, such as the tax- $2 / \operatorname{tax}-4$ channels (Coburn and Bargmann 1996).

In C. elegans, habituation to tactile stimuli (Croll 1975; Chalfie and Sulston 1981; Rankin et al. 1990) and chemosensory stimuli (Ward 1973; Wen et al. 1997) as well as associative learning (Wen et al. 1997; Morrison et al. 1999) and olfactory habituation (Morrison et al. 1999; W.M. Nuttley, S. Harbinder, and D. van der Kooy, in prep.) have been demonstrated. In addition, Colbert and Bargmann $(1995,1997)$ have characterized olfactory adaptation. While genetic components specifically underlying nonassociative learning have yet to be identified, there has been extensive work at the cellular level in identifying pathways in the habituation of the tap-withdrawal response, a form of mechanosensory habituation (Gannon and Rankin 1995; Wicks and Rankin 1996,1997).

We asked if both olfactory adaptation and habituation could be observed in the same paradigm, and if these two processes could be differentiated. After preexposure to very high concentrations (100\%) of DA, worms exhibited a chemotaxis decrement to a point source of DA that did not return to baseline levels following the presentation of various potentially dishabituating stimuli. This DA adaptation was abolished in odr-10 mutants, even though naive chemotaxis remained unaffected, whereas DA adaptation remained intact in odr-1 mutants even though naive chemotaxis to DA decreased. In contrast to $100 \%$ DA preexposure, after preexposure to low DA concentrations (0.001\%), the habituated response could be reinstated to naive chemotaxis levels after presentation of a dishabituating stimulus (centrifugation at $250 \mathrm{~g}$ ). Surprisingly, exposure to intermediate concentrations of DA $(0.01 \%$ and $25 \%)$ did not cause worms to exhibit a response decrement at all. Thus, there

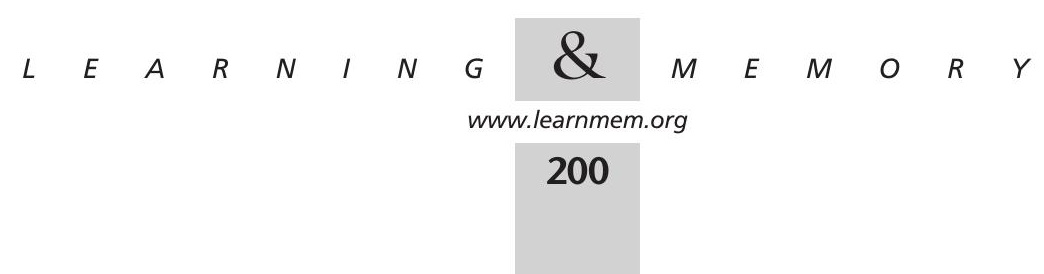


are at least two distinct processes that underlie the observed behavioral response decrement after continuous presentation of an olfactory stimulus, and these two processes, adaptation and habituation, are disassociable in a concentration-dependent manner.

\section{RESULTS}

\section{C. elegans Approaches Different Concentrations of DA in a Dose-Dependent Manner}

To assess behavioral plasticity in C. elegans using DA as an olfactory stimulus, the naive baseline approach to different DA concentrations was established. The animals approached DA in a dose-dependent manner, such that the highest chemotaxis response was elicited at the highest concentrations and as the concentration of DA became more dilute, the chemotaxis index (CI) decreased (Fig. 1B). This corresponds with previous data that show that DA is a significant olfactory attractant over a broad range of concentrations (Bargmann and Horvitz 1991). In addition, it was also noted that the variability in approach to DA was highest at the lowest concentrations of DA.

\section{Preexposure to High Concentrations of DA $(100 \%)$ Leads to a Decrement in Approach that Is Time-Dependent but Volume-Independent}

To test whether worms are capable of showing nonassociative learning at very high concentrations of DA, populations of worms were preexposed for varying exposure times to 5 $\mu 1$ of $100 \%$ DA and their CI was scored after the standard 60 min test period. Naive animals showed high approaches towards a test spot of $100 \%$ DA. A naive group that was starved for $2 \mathrm{hr}$ (there was no noted difference in naive DA approach for animals starved for $30 \mathrm{~min}, 1 \mathrm{hr}$, or $2 \mathrm{hr}$; data not shown) was used as a control for effects of starvation during preexposure time, however there was no significant difference between naive and naive starved groups [t(7) $=1.26, \mathrm{P}>0.05]$. With a $1 \mathrm{hr}$ preexposure, animals showed a significant decrease in approach to DA, and this approach decreased even further after a $2 \mathrm{hr}$ preexposure (Fig. 2A). A one-way ANOVA comparing naive or naive starved animals to preexposed groups with varying exposure times revealed a significant effect of preexposure time $[\mathrm{F}(5,18)=26.0$, $\mathrm{P}<0.05]$. Neuman-Keuls posthoc tests demonstrated that there was no significant effect $(\mathrm{P}>0.05)$ following a $30 \mathrm{~min}$ preexposure, but the $1 \mathrm{hr}$ and $2 \mathrm{hr}$ preexposure treatments yielded CIs that were significantly different from naive starved levels and from each other $(\mathrm{P}<0.05)$. A $3 \mathrm{hr}$ preexposure time was also tested, but despite the substantial decrement in DA approach (data not shown), this lack of response was likely attributable to damage to the animals as seen by general lack of movement and gross clumping at the site of origin on the test plate. Based on these results,
A

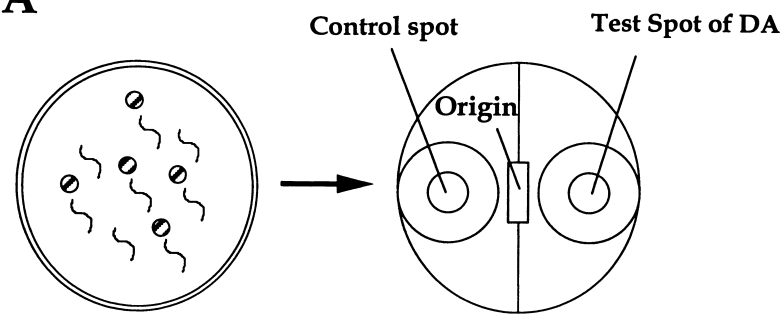

Conditioning plate

\section{$C I=\#$ at test spot $-\#$ at control spot Total on plate}

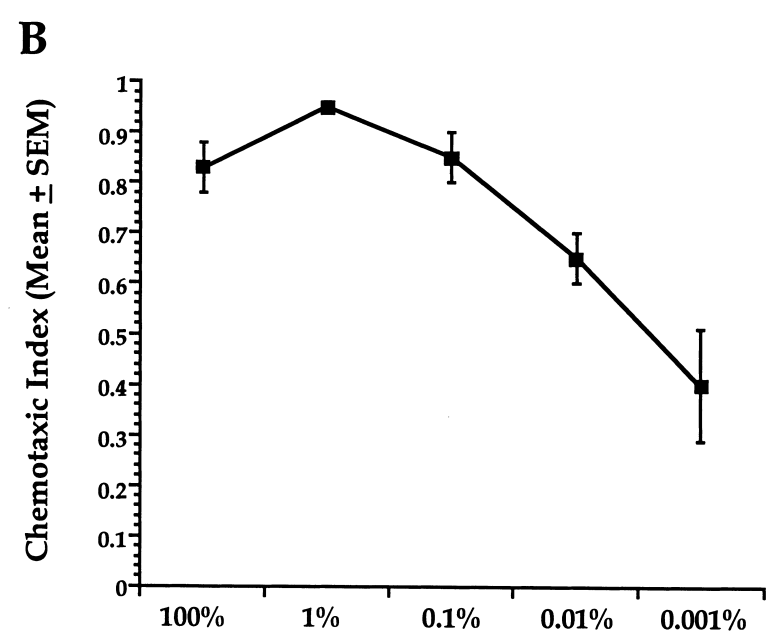

Figure 1 Habituation/adaptation apparatus and dose response to DA. (A) Conditioning agar plates were used for exposing worms to the various concentrations of diacetyl (DA) during training. Circles with hatched bars represent agar plugs, each saturated with $1 \mu$ of DA, and short wavy lines represent aggregates of worms. Following treatment, hundreds of worms were placed at the origin point on a test plate with a $1 \mu \mathrm{l}$ control spot (ethanol, EtOH) and test spot (DA) placed on either end. After a 60 min test period, worms were placed at $4{ }^{\circ} \mathrm{C}$, and later counted to establish a chemotaxis index (Cl) per plate. Each plate consisting of hundreds of worms was considered an $n=1$ each treatment group had an average of $n=4$ per experiment. $(B)$ Worms chemotax to a broad range of DA concentrations. Approach was elicited to a variety of different concentrations of DA, with the highest approach to DA seen at the highest dilutions. $n=4$ for all groups.

the $2 \mathrm{hr}$ preexposure period was chosen to examine the effects of varying the volume of DA on approach. A doseindependent decrease in DA approach was seen, such that regardless of the preexposure volume, the decrement in CI remained constant compared to naive levels (Fig. 2B). A one-way ANOVA comparing naive treatment to preexposed treatments with varying exposure volumes revealed a significant effect of preexposure $[\mathrm{F}(4,15)=7.7, \mathrm{P}<0.05]$. Neu-

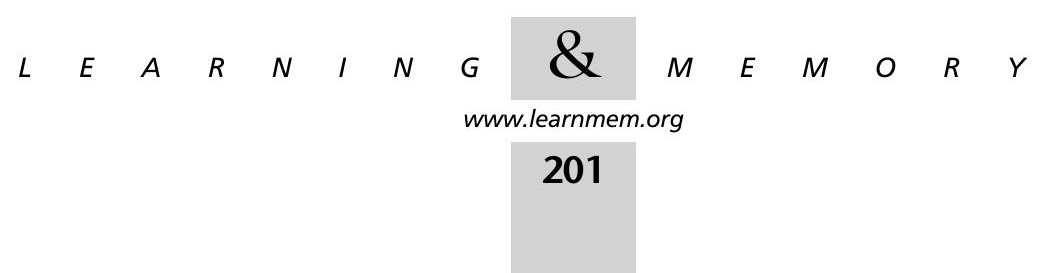



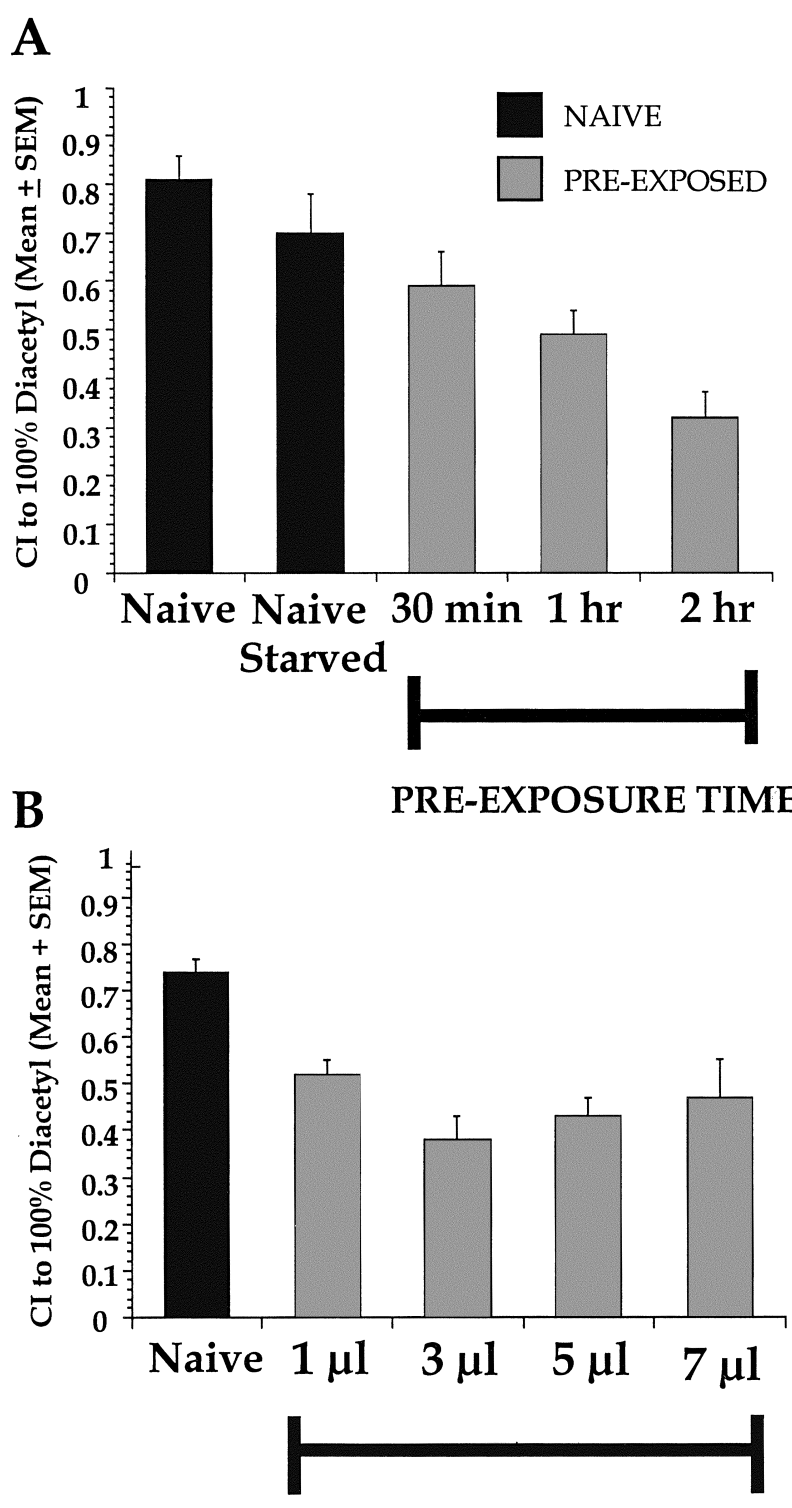

PRE-EXPOSURE VOLUME

Figure 2 Increasing exposure time, but not volume, affects degree of approach decrement after preexposure to $100 \%$ DA. (A) Worms preexposed to $5 \mu \mathrm{l}$ of $100 \%$ DA for $30 \mathrm{~min}$ to $2 \mathrm{hr}$ had significantly lower mean chemotaxis to $100 \%$ DA. The unstarved naive group and the naive group that was starved for $2 \mathrm{hr}$ were not different from each other. After $1 \mathrm{hr}$ of DA exposure, worms showed a significant decrease in approach, but the effect was most pronounced after the $2 \mathrm{hr}$ exposure. $n=4$ for all groups. (B) A $2 \mathrm{hr}$ preexposure time to DA with varying volumes of odorant shows that the approach decrement decreased to $\sim 40 \%$ of naive levels for all groups, regardless of preexposure volume. $n=4$ for all groups.

man-Keuls posthoc tests showed that following preexposure, all groups were significantly different from naive $(\mathrm{P}<0.05)$ and were not significantly different from one another $(\mathrm{P}>0.05)$. Thus, time of exposure but not exposure volume affects the decrement in DA approach.

\section{Exposure to High Concentrations of DA Elicits Adaptation but Not Habituation}

Worms demonstrate a very high CI to a test spot of $100 \%$ DA and can show a decrement in approach following various preexposure times and odorant volumes. Is this decrement in response adaptation, caused by receptor or effector fatigue, or is it due to nonassociative learning that is subject to reversal? By definition, one of the requirements of habituation is a behavioral demonstration of dishabituation. To determine whether the observed decrease in approach behavior represented adaptation or habituation, worms were preexposed to $3 \mu \mathrm{l}$ of $100 \% \mathrm{DA}$ for $2 \mathrm{hr}$, and the dishabituating stimuli varied from centrifugal spins of $100 \mathrm{~g}-$ $1000 \mathrm{~g}$. Regardless of the magnitude of the spin, the stimuli were not sufficient to induce dishabituation of the observed response decrement after $100 \%$ DA preexposure (Fig. 3A). A one-way ANOVA comparing naive, preexposed, and preexposed + spin treatments indicated a significant effect of treatment $[\mathrm{F}(5,17)=8.2, \mathrm{P}<0.05]$. In a Neuman-Keuls posthoc analysis, the CIs of the preexposed and preexposed + spins groups were significantly different from the naive group $(\mathrm{P}<0.05)$, but not from each other $(\mathrm{P}>0.05)$. In a subsequent test we asked if a stronger dishabituating stimulus could cause the response decrement to return to naive levels. After preexposure to $5 \mu \mathrm{l}$ of DA for 1 hr, two different groups of worms were subjected to two different dishabituating treatments: Dishabituation group 1 was given two centrifugal spins at $2000 \mathrm{~g}$ and $30 \mathrm{sec}$ of vortexing, and dishabituation group 2 was spun twice at $100 \mathrm{~g}$ and given a $15 \mathrm{~min}$ cold shock at $4^{\circ} \mathrm{C}$ (Fig. 3B). A one-way ANOVA revealed that animals decreased their approach response after preexposure to $100 \%$ DA $[F(5,52)=22.9, P<0.05]$. Posthoc analysis showed that following conditioning, the preexposed group showed an $\sim 50 \%$ decrease in CI compared to naive $(\mathrm{P}<0.05)$, and that regardless of attempts at dishabituation, this decrement was not reinstated to naive levels. Moreover, the decrement in response seen in the two groups given the dishabituating treatments was not caused by any potentially damaging effects of the treatments, because the naive groups were given equivalent treatments without DA preexposure and these treatments did not affect the high naive baseline CI. To confirm that there was no effect of the dishabituation stimulus on naive CI, a simple comparison of the naive CI to $100 \%$ DA in the manipulated (naive with DH stimulus) worms (Fig. 3B) versus unmanipulated naive animals (i.e., not given a dishabituation stimulus; Fig. 2A), with all other variables such as exposure time and volume kept constant, demonstrated no significant difference [1-way ANOVA, $\mathrm{F}(1,13)=2.82 ; \quad \mathrm{P}>0.05$ or a t-test $\mathrm{t}=-1.67 ; \quad \mathrm{P}>0.05$, $\mathrm{df}=13$ ). Worms left for $3 \mathrm{hr}$ after an equivalent DA preexposure (to spontaneously recover prior to testing) exhibited chemotaxis indices that were not significantly different from naive levels (Fig. 3B, $\mathrm{P}>0.05$ ), indicating that no per-

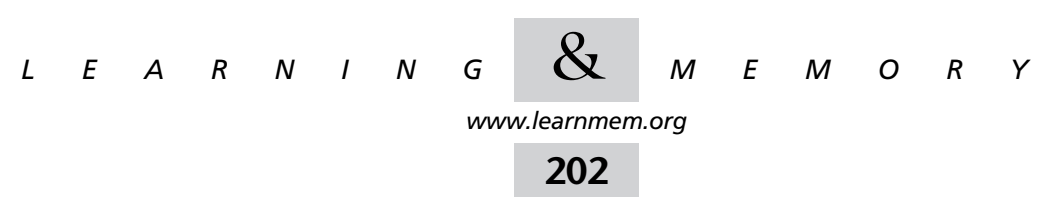




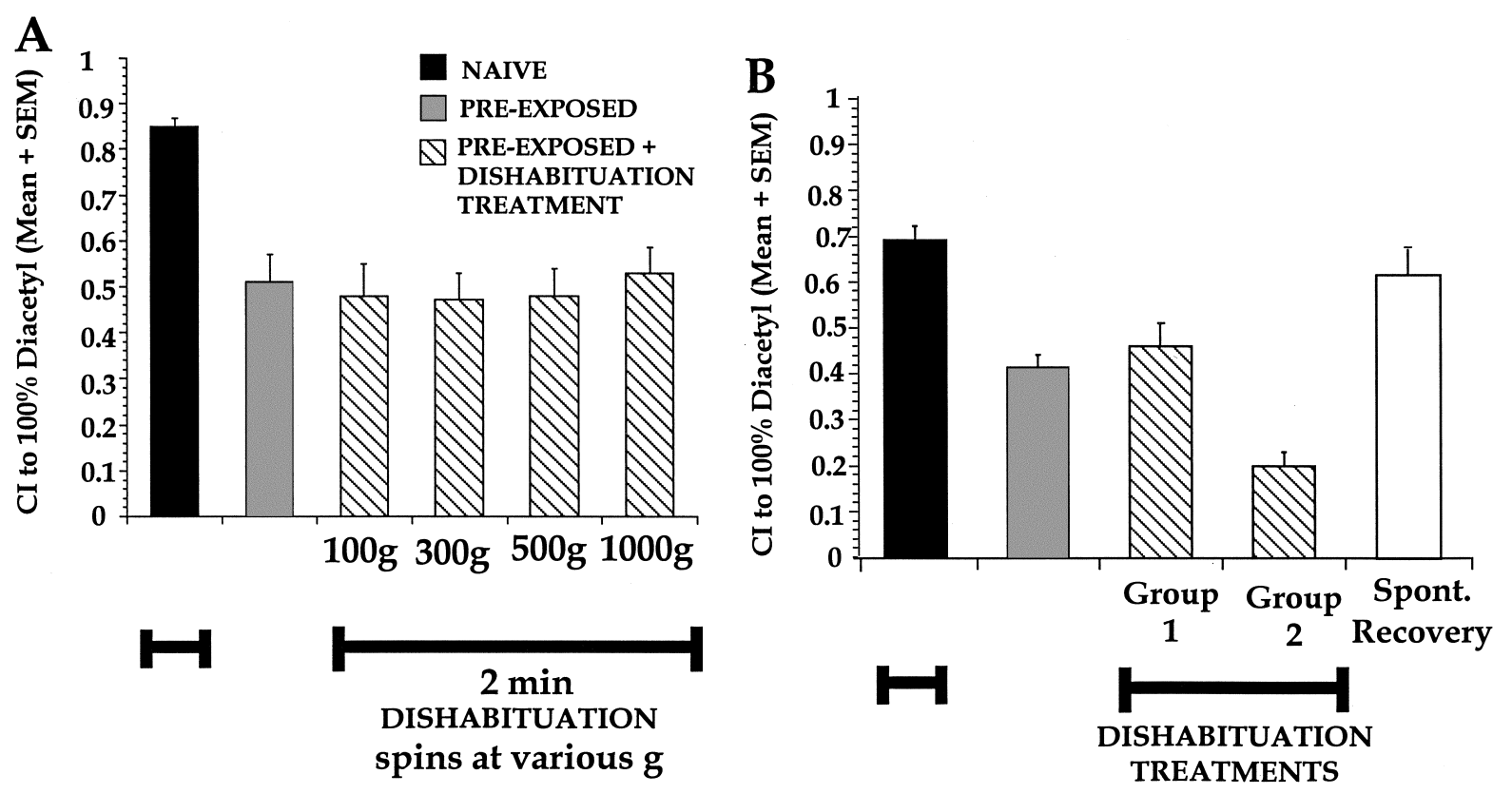

Figure 3 Lack of dishabituation after preexposure to $100 \%$ DA. (A) Preexposure to $3 \mu \mathrm{l}$ of $100 \%$ DA for $2 \mathrm{hr}$ caused worms to exhibit a response decrement to $100 \%$ DA which could not be reversed despite being presented with dishabituating stimuli ranging from 100-1000g spins. The naive group was given a $1000 \mathrm{~g}$ dishabituation treatment without preexposure to DA. All groups preexposed and given dishabituation stimuli were significantly different from the naive group that was not preexposed, but not different from the preexposed group without dishabituation stimuli. $100 \mathrm{~g}=\mathrm{a} 1 \mathrm{~min}$ spin at $100 \mathrm{~g}$ followed by a $2 \mathrm{~min}$ spin at $100 \mathrm{~g} ; 300 \mathrm{~g}=\mathrm{a} 1 \mathrm{~min}$ spin at $300 \mathrm{~g}$ followed by a $2 \mathrm{~min}$ spin at $300 \mathrm{~g} ; 500 \mathrm{~g}=\mathrm{a} 1 \mathrm{~min}$ spin at $500 \mathrm{~g}$ followed by a $2 \mathrm{~min}$ spin at $500 \mathrm{~g} ; 1000 \mathrm{~g}=\mathrm{a} 1 \mathrm{~min}$ spin at $1000 \mathrm{~g}$ followed by a 2 min spin at 1000g. $n=4$ for all groups, except $n=3$ for naive. (B) Worms preexposed to $5 \mu \mathrm{l}$ of $100 \%$ DA for 1 hr do not show a dishabituated response to $100 \%$ DA following different dishabituating treatments. Naive group given dishabituating stimulus without DA preexposure. Preexpose $=$ preexposed without dishabituating treatments; Group $1=$ preexposure $+2 \times 2000 \mathrm{~g}$ spins $(3 \mathrm{~min}$ total $)+30 \mathrm{sec}$ vortex; Group $2=$ preexposure + cold shock treatment of $4^{\circ} \mathrm{C}$ for $15 \mathrm{~min}$, followed by a $1 \mathrm{~min}$ spin at $100 \mathrm{~g}$; Spont. Recovery = spontaneous recovery after 3 hr. $n=20,18,4,8$, and 4, respectively, from left to right.

manent damage was caused by $100 \%$ DA preexposure as their CIs were restored to prior naive baseline levels. While there is some evidence demonstrating that aromatic ketones, such as DA cause molecular modifications of cellular organelles such as mitochondria and peroxisomes (Adak et al. 1996; Eriksson et al. 1998), the present data suggests that the one hr and $2 \mathrm{hr}$ exposure time of DA exposures utilized were not damaging. Thus, preexposure to $100 \% \mathrm{DA}$ results in adaptation because dishabituation is not revealed despite the potency of the dishabituating stimuli (see below).

\section{Exposure to Intermediate Concentrations of DA (25\% and $0.01 \%$ ) Leads to No Response Decrement in DA Approach}

Since exposure to $100 \%$ DA leads to adaptation, might DA concentrations $<100 \%$ elicit a similar response decrement or a different behavioral response? Preexposure to $25 \%$ DA was not sufficient to cause a decrement in approach to the same $25 \%$ DA stimulus regardless of exposure time or amount of DAl present (Fig. 4A). The dishabituating stimulus of two strong centrifugal spins at $500 \mathrm{~g}$ did not affect approach to DA in either the naive or preexposed + spin groups. A one-way ANOVA examining a 90 min preexpo- sure to $2 \mu \mathrm{l}$ of $25 \% \mathrm{DA}$ in naive, preexposed, and preexposed + spin groups revealed no significant effect of treatments $[\mathrm{F}(2,8)=2.3, \mathrm{P}>0.05]$. Similarly, a $2 \mathrm{hr}$ preexposure to $3 \mu \mathrm{l}$ of $25 \%$ DA yielded no significant effect of treatment $[\mathrm{F}(2,9)=2.1, \mathrm{P}>0.05]$. Because the absolute approach to $25 \% \mathrm{DA}$ was so high, the effects of preexposure to a concentration of DA that was lower than 25\% DA by $\sim 3$ orders of magnitude $(0.01 \%$ DA) was examined. Preexposure to $5 \mu \mathrm{l} 0.01 \% \mathrm{DA}$ for $15 \mathrm{~min}$ was not sufficient induce a decrement in CI to $0.01 \%$ DA. A one-way ANOVA comparing naive, preexposed, and preexposed + spin groups revealed no significant effects of treatment $[\mathrm{F}(2,15))=0.2$, $\mathrm{P}>0.05$ ] (Fig. 4B). Preexposure to the same concentration of DA for a longer $60 \mathrm{~min}$ duration, however, showed a trend toward a decrease in CI, but a one-way ANOVA revealed no significant difference among these groups $[F(2,11))=2.63, P>0.05]$. In subsequent attempts to see if an even longer preexposure time of 90 min would drive the $\mathrm{CI}$ down even further, there were no significant reductions from the naive $\mathrm{CI}[\mathrm{F}(2,12)=1.97, \mathrm{P}>0.05$, data not shown] Despite variations in length and volume of preexposure to the odorant, intermediate concentrations of DA $(<100 \%$ and $\geqslant 0.01 \%$ ) do not produce decremental responses.

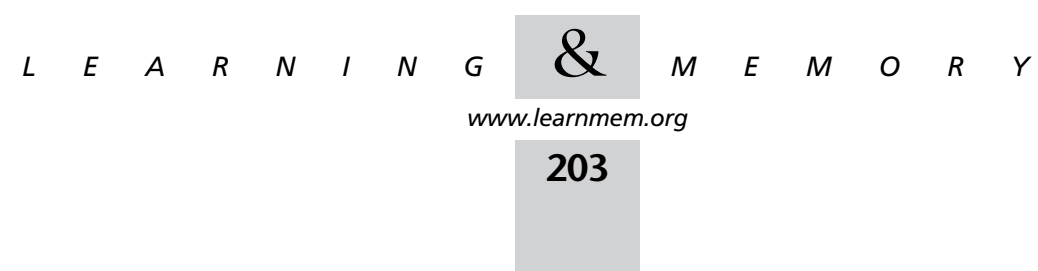




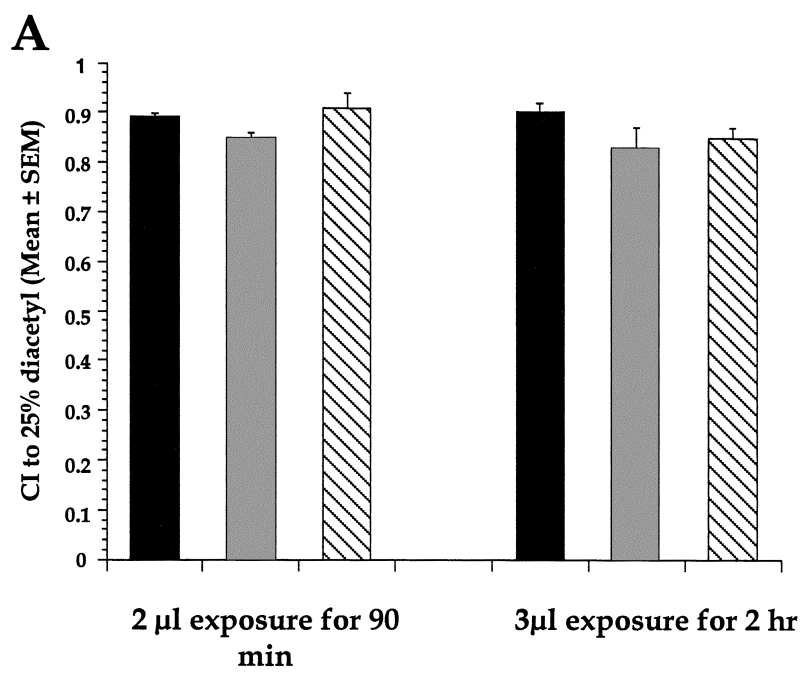

B

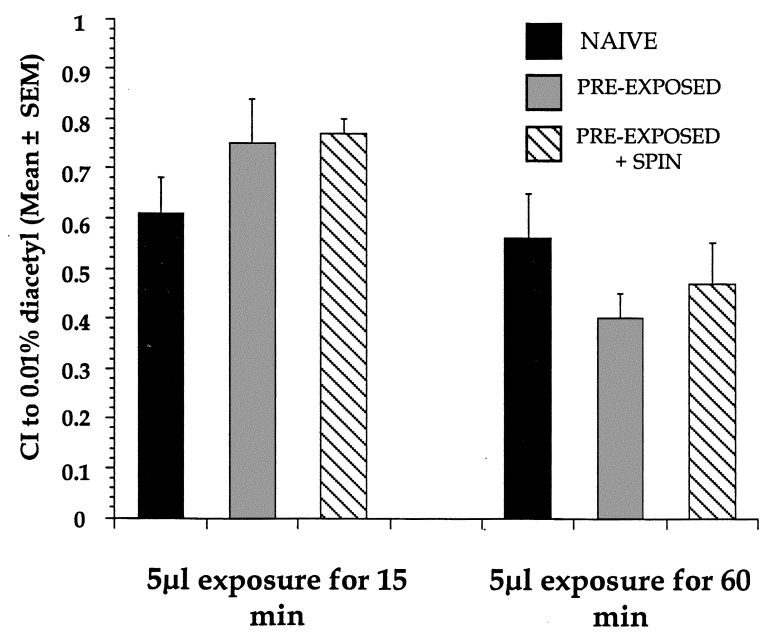

Figure 4 No response decrement seen after exposure to intermediate DA concentrations ( $25 \%, 0.01 \%)$. (A) Preexposures to $2 \mu \mathrm{l}$ of $25 \%$ DA for $90 \mathrm{~min}$ or $3 \mu \mathrm{l}$ of $25 \%$ DA for $2 \mathrm{hr}$ were not sufficient to elicit a response decrement to $25 \%$ DA in wild-type animals. The dishabituating stimulus was a 1 min centrifugation at $500 \mathrm{~g}$, followed by a $2 \min 500 \mathrm{~g}$ spin. $n=4$ for all, except $n=3$ for naive $2 \mu \mathrm{l} / 90$ min exposure group. (B) A $5 \mu \mathrm{l}$ preexposure to $0.01 \%$ DA did not cause a decreased chemotaxis approach to $0.01 \%$ DA despite varying the time of exposure from 15-60 min. The dishabituating stimulus was a 1 min centrifugation at $500 \mathrm{~g}$, followed by a $2 \min 500 g$ spin. $n=4$ for all 15 min groups, and $n=3$ for all 60 min groups.

\section{Preexposure to Low Concentrations of DA $(0.001 \%)$ Favors Nonassociative Learning}

The effects of preexposure to low concentrations of DA $(0.001 \%)$ were observed and compared with preexposure to intermediate DA (25\% and $0.01 \%)$ concentrations to determine whether there would be a similar lack of response decrement as seen after $25 \%$ or $0.01 \%$ DA preexposure. In an analysis of all experiments, preexposure treatment (habituation) was compared to preexposure + spin treatment (dishabituation) and naive scores. Worms exhibited a 25\% decrease in approach to $0.001 \%$ DA after $0.001 \%$ DA preexposure, and after the dishabituation treatment their CI returned to naive levels (Fig. 5). Thus, unlike the response decrement of adapted worms, the response decrement of the habituated worms demonstrated the ability to return to baseline levels immediately after presentation of a dishabituating stimulus. In addition to dishabituation, the habituated group was also able to demonstrate spontaneous recovery over time, even without the presentation of a dishabituating stimulus. When left to recover for $2 \mathrm{hr}$ after preexposure, DA approach recovered spontaneously back to naive and dishabituated levels. A one-way ANOVA comparing naive, habituated, dishabituated, and spontaneous recovery treatments showed that there was a significant effect of treatment $[\mathrm{F}(2,282)=10.0, \mathrm{P}<0.05]$ and posthoc tests revealed that the habituation treatment was significantly different from the naive, dishabituation, and spontaneous recovery treatments $(\mathrm{P}<0.05)$, which were not significantly different from each other $(\mathrm{P}>0.05)$. Thus, preexposue to low concentrations of DA leads to the nonassociative learning process of habituation, as demonstrated by the ability of pre-

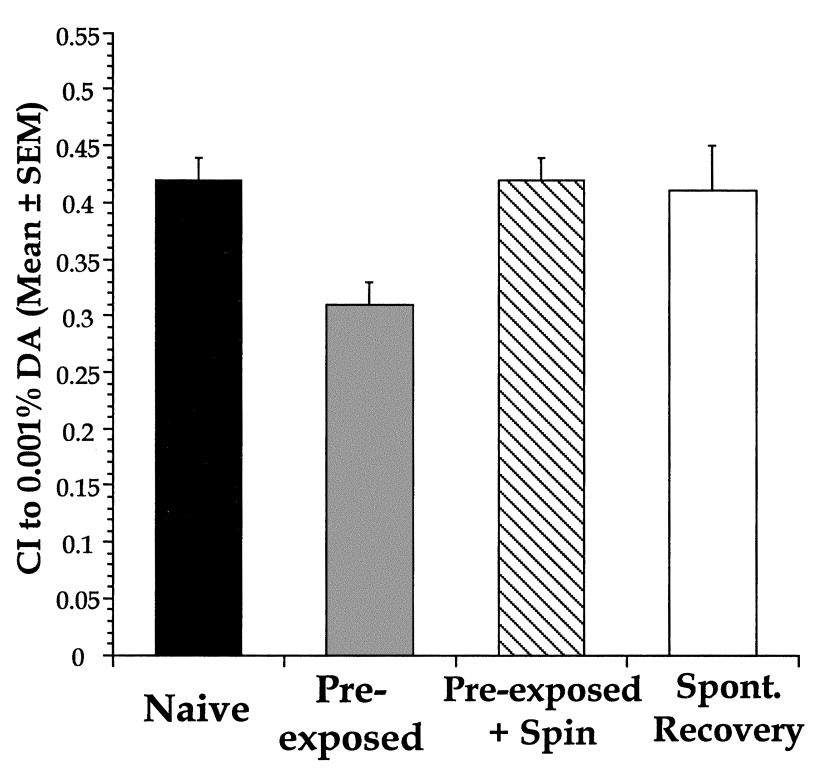

Figure 5 Nonassociative learning occurs after preexposure to low concentrations of DA $(0.001 \%)$. Preexposure to $0.001 \%$ DA leads to habituation. Worms preexposed for $15 \mathrm{~min}$ to $5 \mu \mathrm{l}$ of $0.001 \%$ DA showed a $25 \%$ response decrement when tested to $0.001 \%$ DA compared with naive values. Preexposed + spin groups centrifuged twice for a total of $3 \mathrm{~min}$ at $250 \mathrm{~g}$ exhibited a recovery of chemotaxic approach to $0.001 \%$ DA to the levels seen in the naive or spontaneous recovery groups. All naive worms (Naive) were given the dishabituating treatment (spin) without DA preexposure. $n$ $=100,106,103$, and 10, respectively, from left to right.

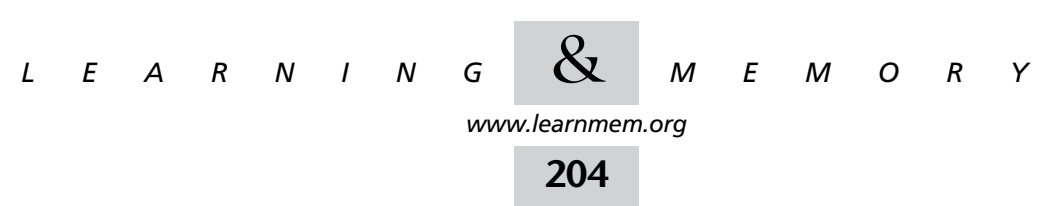


exposed worms to dishabituate by returning to naive CI levels.

\section{Adaptation and Nonassociative Learning Are Distinct Processes that Are Separable in a Concentration-Dependent Manner}

Preexposure to both $100 \%$ DA and $0.001 \%$ DA elicited a response decrement, however the decreased response after preexposure to $0.001 \%$ DA could be dishabituated, whereas the decreased response after 100\% DA preexposure could not. In addition, preexposure to intermediate concentrations of DA did not elicit any significant response decrement. To test if there is a significant difference between the various concentrations $(100 \%, 25 \%, 0.01 \%$, and $0.001 \% \mathrm{DA})$ in response to treatments (naive, preexposed/habituation, and preexposed + spin/dishabituation), a two-way ANOVA was carried out. This showed a significant interaction of Concentration $\times$ Treatment $[\mathrm{F}(6,432)=5.27, \mathrm{P}<0.05]$, demonstrating that the effects of the treatments are different at differing DA concentrations.

\section{DA Adaptation Requires odr-10 but Not odr-1}

Wild-type N2 worms demonstrate adaptation after being preexposed to $100 \% \mathrm{DA}$, however the pathways that mediate this response are unknown. Approaches to high concentrations of DA are likely mediated by a low-affinity DA receptor on the AWC neuron, because animals lacking the $o d r-10$ gene or function of the AWA neuron still approach high DA concentrations (Chou et al. 1997; Sengupta et al. 1996), but animals with odr-1 -induced deficiencies in AWC function show impaired chemotaxis to high DA concentrations (Bargmann et al. 1993). Thus, mutations that affect the function of the two putative DA-sensing primary chemosensory neurons [odr-10, which is the high-affinity DA receptor in AWA (Sengupta et al. 1996), and odr-1, which affects AWC function (Bargmann et al. 1993)] were tested for their adaptation responses to $100 \%$ DA. Wild-type, odr-10, and $o d r-1$ strains were preexposed for $60 \mathrm{~min}$ to $5 \mu \mathrm{l}$ of $100 \%$ $\mathrm{DA}$, and tested for their responses to $100 \% \mathrm{DA}, 0.1 \% \mathrm{DA}$, and $1 \%$ benzaldehyde (Fig. 6). A three-way ANOVA comparing Strain $\times$ Olfactory Stimulus $\times$ Treatment demonstrated a significant interaction among these variables $[\mathrm{F}(16,272)=$ $27.52, \mathrm{P}<0.05]$. We then proceeded to carry out individual analyses within the wild-type strain, and then across strains.

As expected, wild-type worms preexposed to $100 \%$ DA and tested to $100 \%$ or $0.1 \%$ DA demonstrated a decrease in CI compared to their respective wild-type naive values. A two-way ANOVA revealed a significant interaction of treatments with olfactory stimuli $[\mathrm{F}(4,125)=9.84, \mathrm{P}<0.05]$ in the wild-type animals, and posthoc analyses confirm that $100 \%$ DA preexposure caused a significant $(\sim 50 \%)$ reduction in DA approach to $100 \%$ DA $(\mathrm{P}<0.05)$. Wild-type worms preexposed to $100 \% \mathrm{DA}$ and tested to $0.1 \%$ showed an even larger decrement in approach $(\sim 75 \%$ decrease, $\mathrm{P}<0.05)$. Preexposed wild-type animals tested to either $100 \%$ DA or $0.1 \%$ DA did not display any signs of dishabituation after being spun twice for a total of $3 \mathrm{~min}$ (preexposure + spin treatment). Wild-type approach to BZ was high, although there did appear to be a small yet significant decrease after DA preexposure (Neuman-Keuls posthoc test, $\mathrm{P}<0.05$ ).

Worms of the $o d r-10$ strain exhibited high naive chemotaxis to $100 \% \mathrm{DA}$ and $1 \% \mathrm{BZ}$, with approach to $0.1 \% \mathrm{DA}$ almost completely eliminated as shown previously by Sengupta et al. (1996). Interestingly, testing to $100 \%$ DA revealed that unlike wild-type, odr-10 worms did not demonstrate any significant decrement in response to DA after a 60 min preexposure to $100 \%$ DA. A three-way ANOVA of $o d r$ 10 and wild-type worms comparing Strain $\times$ Treatment $\times$ Olfactory Stimulus showed a significant three-way interaction $[\mathrm{F}(4,197)=7.69, \mathrm{P}<0.05]$; closer comparison of Strain with Treatment in response to $100 \%$ DA also showed a significant interaction $[\mathrm{F}(2,68)=5.87, \mathrm{P}<0.05)$. Post-hoc comparisons of wild-type and $o d r-10$ groups pre-exposed and tested to $100 \%$ DA yielded significant differences between wild-type Naive and Pre-exposed or Pre-exposed + Spin groups $(\mathrm{P}<0.05)$ [with no difference between Pre-exposed and Pre-exposed + spin $(\mathrm{P}>0.05)]$, while no significant differences between $o d r-10$ Naive and Pre-exposed or Pre-exposed + Spin groups $(\mathrm{P}>0.05)$ were observed. Thus, unlike wild-type, odr-10 worms did not adapt.

After preexposure to $100 \%$ DA, the odr-1 worms demonstrated adaptation of the chemotaxis response when tested to DA, although their naive CI to both $100 \%$ DA and $0.1 \%$ DA were lower than the naive CI of wild-type worms. An analysis of the wild-type and odr-1 strains over Treatment (Naive, Pre-exposed, and Pre-exposed + Spin) and with Olfactory Stimulus revealed a significant three-way interaction $[\mathrm{F}(4,200)=4.03, \mathrm{P}<0.05)$. The odr-1 worms displayed a compromised naive $\mathrm{CI}$ to $100 \%$ and $0.1 \% \mathrm{DA}$ (Neuman-Keuls post-hoc tests, $\mathrm{P}<0.05$, significantly different from the comparable wild-type values). In addition, the odr-1 worms displayed a Naive CI to $100 \%$ DA that was lower than the Naive CI to $0.1 \%$ DA $[\mathrm{t}(16)=2.3, \mathrm{P}<0.05]$, indicating that in $o d r-1$ worms, the naive CI to DA increased as the concentration of DA decreased from $100 \%$ to $0.1 \%$ (this provides further support for the hypothesis of a lowaffinity DA receptor on AWC mediating high-concentration DA responses). Despite the lower baseline CIs, however, after $100 \%$ DA preexposure the odr-1 worms still demonstrated an $\sim 50 \%$ and $\sim 80 \%$ decreased CI when tested to $100 \% \mathrm{DA}$, and $0.1 \% \mathrm{DA}$, respectively $(\mathrm{P}<0.05)$. This decrement in the odr-1 worms could not be dishabituated with a dishabituating stimulus (same as above for wild-type and odr-10 worms). An almost complete lack of BZ approach in naive $o d r-1$ worms confirms the prior classification of the $o d r-1$ strain as particularly deficient in AWC function (Barg-

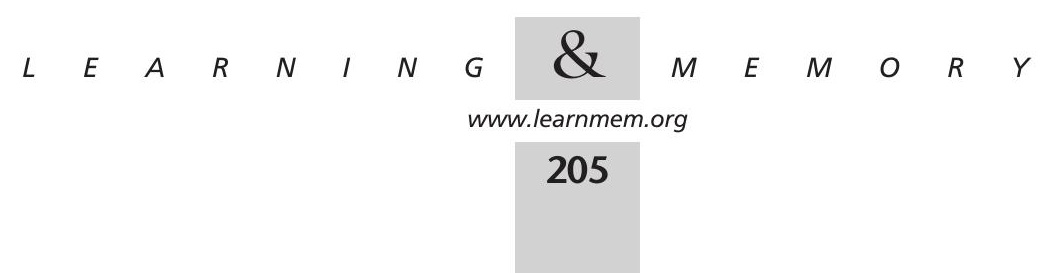


A
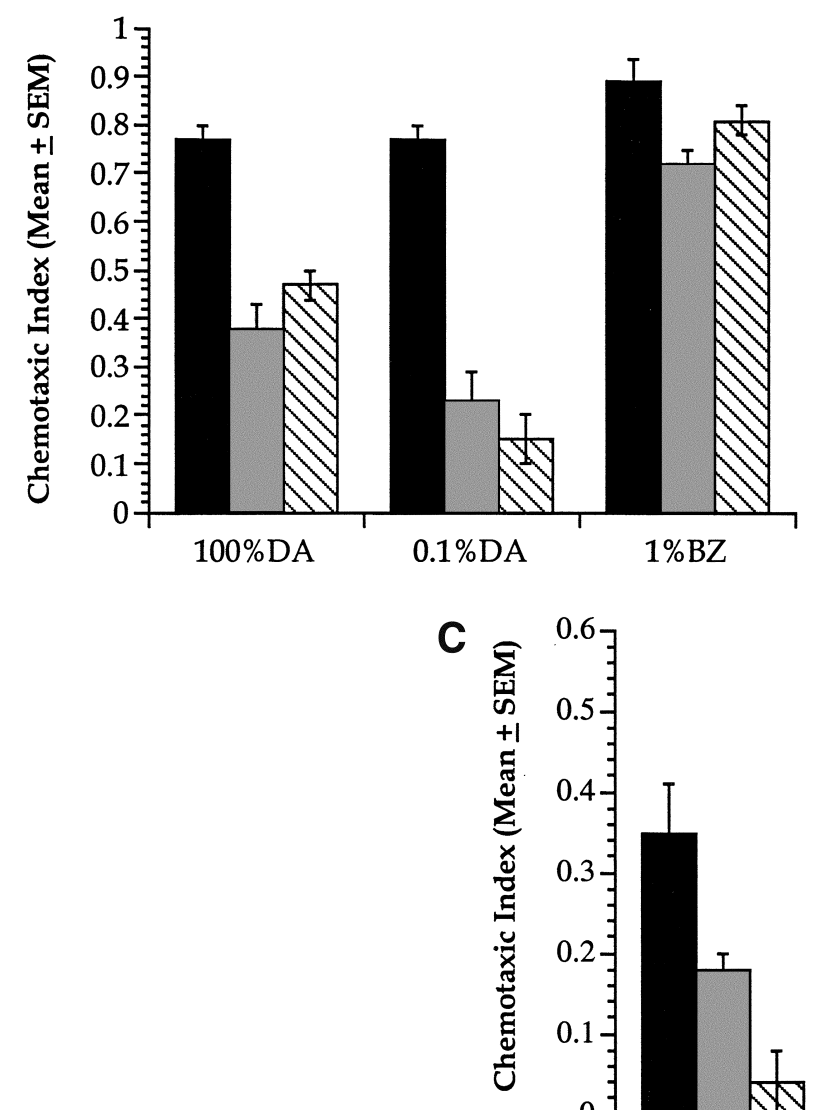

B

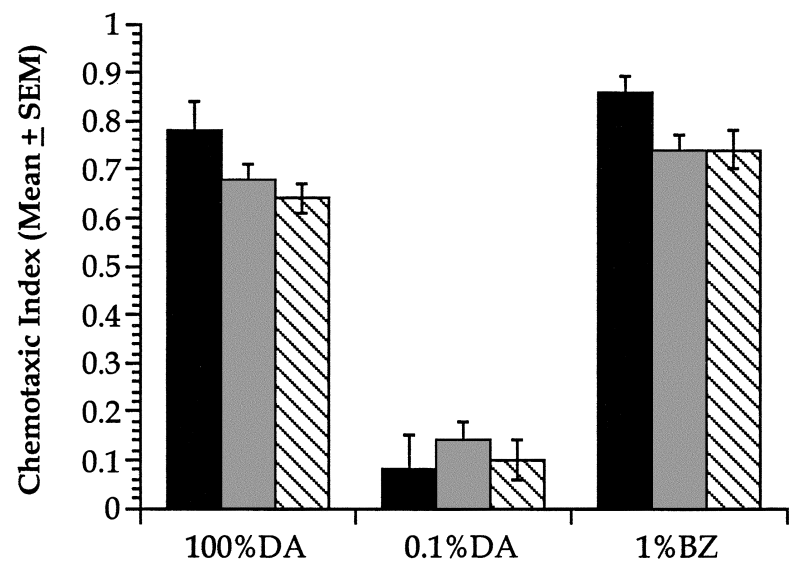

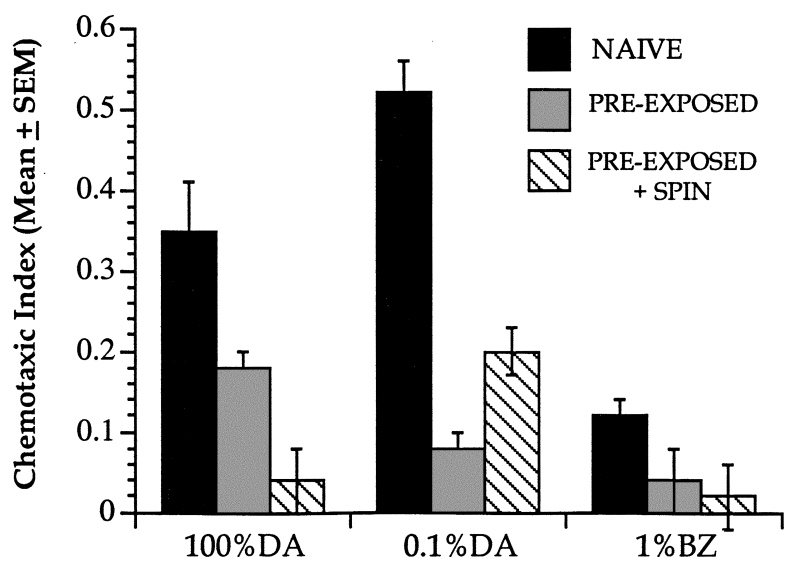

Figure 6 Adaptation is odr-10 dependent, but odr-1 independent. Wild-type, odr-10, and odr-1 worms were preexposed to $5 \mu$ of $100 \%$ DA for $60 \mathrm{~min}$ and tested to $100 \%$ DA, $0.1 \%$ DA, or $1 \%$ BZ. Wild-type worms displayed a $50 \%$ decrease in CI to $100 \%$ DA and a $75 \%$ decrease in $\mathrm{Cl}$ to $0.1 \%$ DA (compared with their respective naive values), but the $\mathrm{Cl}$ to $\mathrm{BZ}$ did not show a similar decrement after $100 \% \mathrm{DA}$ preexposure. Preexposed group $=$ preexposure with a gentle wash and settled in a tube; Preexposed + Spin $=$ preexposed and spun at $500 g$ for $1 \mathrm{~min}$ followed by another $500 \mathrm{~g}$ spin for $2 \mathrm{~min}$. Within each strain, there was no difference between preexposed or preexposed + spin treatments. odr-10 animals, which showed a nearly zero response to $0.1 \%$ DA, did not demonstrate DA adaptation after $100 \%$ DA preexposure and testing, and did not show any difference in BZ approach compared to wild-type. odr-1 animals displayed lower baseline Cls to $100 \%$ DA and $0.1 \%$ DA and no response to BZ, yet still demonstrated DA adaptation to $100 \%$ and $0.1 \%$ DA after $100 \%$ DA preexposure. Respectively from left to right: for Wild-type $100 \% \mathrm{DA}, n=17,16,15 ; 0.1 \% \mathrm{DA}, n=17,18,15 ; 1 \% \mathrm{BZ}, n=12,11,14$. For odr-10 100\% DA, $n=9,9,8 ; 0.1 \% \mathrm{DA}, n=9,9,10 ; 1 \% \mathrm{BZ} n=9$ for all groups. For odr-1 100\% DA, $n=7,6,8 ; 0.1 \% \mathrm{DA}, n=11$, 12,$12 ; 1 \% \mathrm{BZ}, n=11,9,8$.

mann et al. 1993). Within the wild-type and odr-1 strains, a simple comparison of response to $0.1 \%$ DA (the concentration where wild-type and $o d r-1$ worms had naive CI values that were most similar) in a two-way ANOVA investigating Strain and Treatment interactions showed a significant interaction $[\mathrm{F}(2,78)=5.20, \mathrm{P}<0.05]$. A closer analysis of Treatment using Neuman-Keuls posthoc tests demonstrated that there was a significant difference between wild-type and $o d r-1$ naive treatments $(\mathrm{P}>0.05)$, but no significant difference between the wild-type preexposed or preexposed + spin treatments compared to the odr-1 preexposed or preexposed + spin treatments $(\mathrm{P}>0.05)$. The preexposed or preexposed + spin treatments were not different from each other in either the wild-type or odr-1 strains $(\mathrm{P}>0.05)$.
The lower baseline approaches to DA and $\mathrm{BZ}$ in odr-1 may suggest a broad inability to sense volatile odorants or to chemotax normally. We tested the possibility that the odr-1 strain had generalized motor or sensory deficits by observing the chemotaxis response to the AWA-sensed odorant, pyrazine (Bargmann and Horvitz 1991). Naive approach to $100 \mathrm{mg} / \mathrm{ml}$ and $20 \mathrm{mg} / \mathrm{ml}$ of pyrazine in the odr-1 strain did not differ significantly from the wild-type naive approach [two-way ANOVA $\mathrm{F}(1,12)=0.13, \mathrm{P}>0.05$, wild-type naive CI to $100 \mathrm{mg} / \mathrm{ml}=0.78 \pm 0.09$, wild-type naive CI to 20 $\mathrm{mg} / \mathrm{ml}=0.88 \pm 0.01, o d r-1$ naive CI to $100 \mathrm{mg} / \mathrm{ml}=0.78 \pm$ $0.05, o d r-1$ naive CI to $20 \mathrm{mg} / \mathrm{ml}=0.84 \pm 0.01, n=4$ for all groups, respectively].

Overall, these experiments demonstrate that adapta-

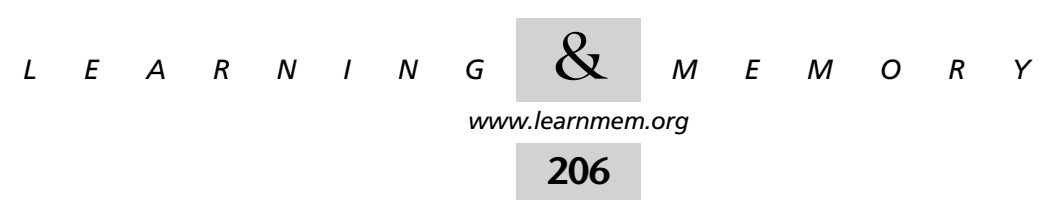


tion to DA is mediated through an odr-1-independent pathway, and that lack of $o d r-10$ prevents adaptation.

\section{DISCUSSION}

The decrement in chemotaxic approach subsequent to continuous presentation of the volatile attractant DA can be accounted for by two distinct forms of olfactory plasticity depending on the odorant concentration. After preexposure to high DA concentrations (100\%), the observed chemotaxis decrement could not be reversed to naive approach levels despite the immediate presentation of strong dishabituating stimuli, and thus the cause of such a decrement is likely due to a form of sensory or receptor fatigue yielding behavioral adaptation of the response. After preexposure to intermediate DA concentrations (0.01\%-25\%) there was no observable decrease in DA chemotaxis. After preexposure to low concentrations of DA $(0.001 \%)$ the behavioral plasticity observed was due to nonassociative learning: The decreased response was a result of habituation, as a strong stimulus was sufficient to induce dishabituation. On a mechanistic level, lack of the ODR-10 high-affinity DA receptor on the AWA neuron prevented DA adaptation without affecting high baseline naive CI to $100 \%$ DA, and diminishing AWC primary chemosensory function by eliminating the $o d r-1$ gene reduced baseline naive $\mathrm{CI}$, but did not prevent normal DA adaptation.

To account for the concentration-specific effects of DA preexposure, the following three processes are postulated to underlie the observed behavioral responses: adaptation (receptor fatigue), habituation, and the theoretical presence of a sensitization process (which would result in an increase above baseline response) (Fig. 7). Although we have no clear evidence that sensitization occurs within the paramaters of our paradigm, we postulate that it may be an underlying mechanism that plays a role in the observed responses and that given the appropriate conditions (such as those suggested by Groves and Thompson 1970) a sensitization response may be observed.

Whereas $100 \%$ DA is sensed by both low-affinity and high-affinity receptors, our model predicts that naive approach to $100 \%$ DA requires a low-affinity DA receptor acting through $o d r-1$ signaling, while $o d r-10$ may account for a portion of $100 \%$ DA approach in the absence of odr-1 function (Figs. 6 and 7A). After preexposure to $100 \%$ DA, we suggest that excessive activation of the high-affinity ODR-10 DA receptor causes ODR-10 downstream targets to interact with the AWC low-affinity DA receptor pathway leading to its down-regulation and causing adaptation of the approach to $100 \%$ DA. This interaction between the two primary chemosensory neurons may be through common downstream neuronal targets such as the AIY or AIZ interneurons (White et al. 1986). Evidence of such cross talk between AWA and AWC is supported by studies which demonstrate that butanone (an AWC-sensed odorant) sensi- tivity is increased slightly in odr-10 mutants (Sengupta et al. 1996). After preexposure to $100 \% \mathrm{DA}$, approach to this concentration of odorant can only be reinstated to naive levels after sufficient time for up-regulation (recovery) of the AWC-mediated approach pathway. Despite the strong intensity of the stimulus, we hypothesize that sensitization is not favored to occur at this concentration as cellular fatigue prevents any modulation due to nonassociative learning.

At intermediate DA concentrations (0.01\%-25\%) the model predicts that there is less activation of the AWC lowaffinity receptor as well as strong activation of the highaffinity ODR-10 receptor (Fig. 7B). Whereas the stimulation of the ODR-10 receptor is still strong, it is not as great as with $100 \%$ DA preexposure so that adaptation is less and/or sensitization is greater. Thus, there is less cellular fatigue/ adaptation and now both sensitization and adaptation are favored equally, which leads to a competition between the two processes. Although the theoretical process of sensitization is favored due to the strength of the preexposure stimulus (high stimulus intensity), adaptation is also occurring. Any potential decrement in response that would be caused by adaptation is opposed by the theoretical sensitization that is attempting to facilitate the response. Hence, the overall observed behavioral response after DA preexposure to such intermediate concentrations is no change in the chemotaxis approach. It must be noted, however, that only two different concentrations of DA were tested within the intermediate DA concentrations. It is therefore possible that the ideal conditions to observe a net behavioral response lies somewhere in between the tested concentrations. This remains an unlikely possiblity as the assessed concentrations ( $25 \%$ and $0.01 \% \mathrm{DA})$ covered more than two orders of magnitude without any observable difference of treatments. An alternative possibility which remains is that neither the adaptation nor the sensitization processes are activated over the range of intermediate DA concentrations, and that the complete absence of these processes underlies the lack of behavioral response after DA preexposure. This possibility seems unlikely, however, because even at lower DA concentrations (0.001\%) plastic behavioral responses are observed, suggesting that at the intermediate DA concentrations the plastic processes mediating such behavioral responses may be masked by competition.

At the lowest DA concentrations (0.001\%) only the ODR-10 receptor is stimulated without activation of the AWC low-affinity DA receptor, and our model thus predicts that any possible AWC-mediated adaptation (through a downstream interaction of the AWA and AWC pathways) is eliminated. Therefore the lack of AWC activation and stimulation of only odr-10 at these low DA concentrations allows the nonassociative learning process of habituation to occur (Fig. 7C). At low stimulus intensities, Groves and Thompson (1970) would predict that habituation should

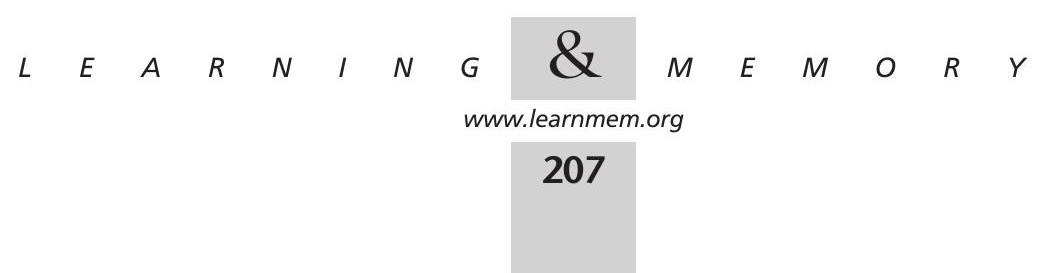




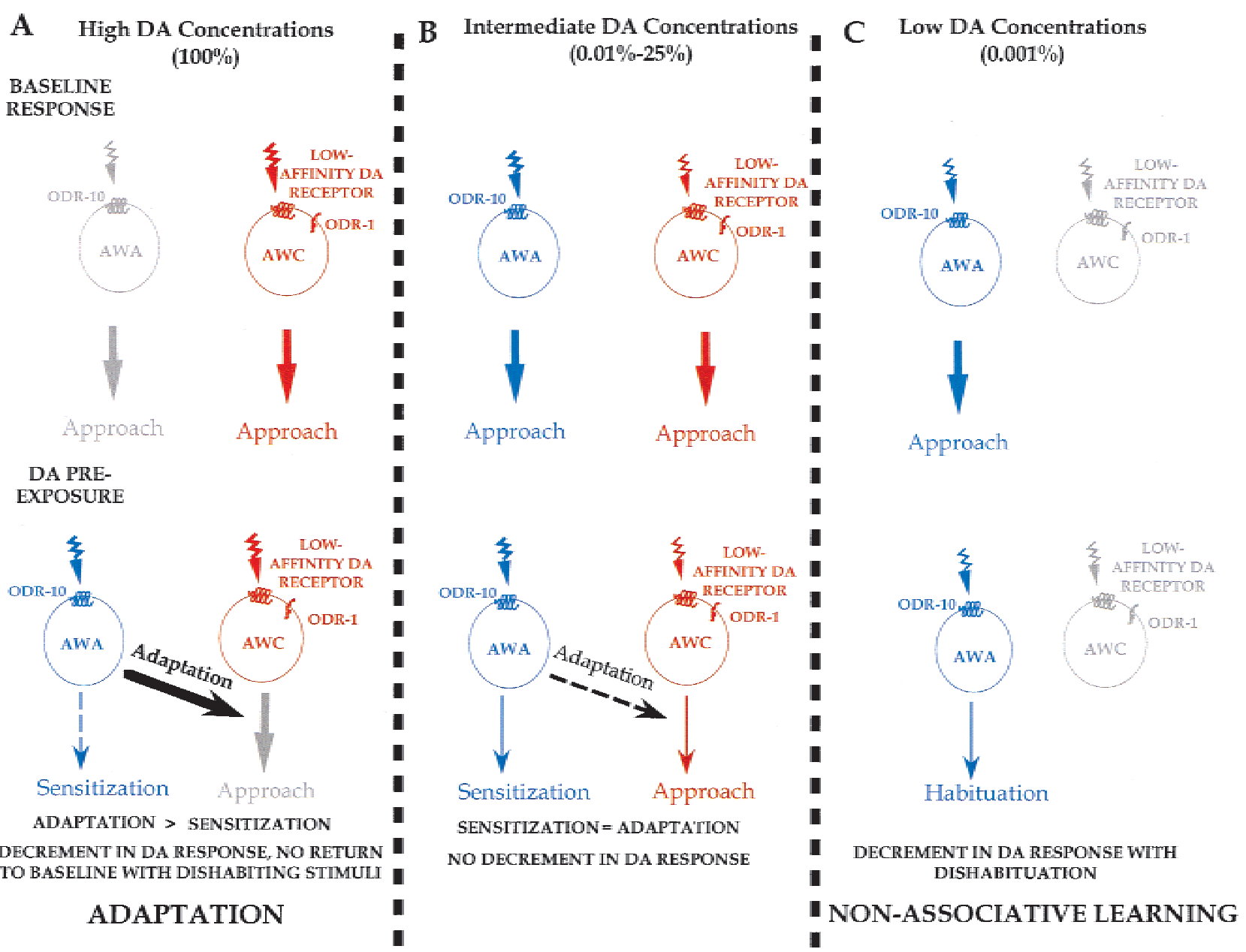

Figure 7 Adaptation and habituation are separate processes that affect the behavioral response after preexposure to DA. (A) Under baseline conditions, the putative low-affinity DA receptor on AWC mediates a portion of the naive approach to 100\% DA via an odr-1-dependent pathway. Although the ODR-10 receptor on AWA is not necessary for naive approach to DA, it can account for a portion of the response to $100 \%$ DA in the absence of AWC function. Preexposure to $100 \%$ DA causes excessive activation of the high-affinity odr-10 receptor on AWA which leads to a down regulation of the AWC mediated approach pathway causing adaptation. This interaction between AWC and AWA may be via a common downstream neuronal target. Lack of odr-1 in the AWC neuron partially eliminates baseline approach to $100 \%$ DA, but does not prevent the odr-10-mediated adaptation. Any sensitization that may be occurring in AWA is overshadowed by the adaptation. (B) Preexposure to intermediate DA concentrations causes less stimulation of the low-affinity DA receptor on AWC and strong stimulation of the ODR-10 DA receptor (but less stimulation than with $100 \%$ DA so that adaptation is less and/or sensitization is greater). This leads to the processes of adaptation and sensitization to be equivalently favored and the competition between these two opposing processes results in no net behavioral decrement in DA response. (C) Preexposure to low DA concentrations causes stimulation of only the ODR-10 receptor on AWA (and no activation of the low-affinity DA receptor on AWC), eliminating any possible response adaptation. This results in the nonassociative learning processes of habituation, dependent on AWA function. Gray = inactive neurons; blue = activated AWA neuron; red $=$ activated AWC neuron.

occur after $0.001 \%$ DA preexposure; this is observed in the worms that demonstrate a habituated response after DA preexposure that can be dishabituated with a sufficiently strong stimulus.

Other experiments in Drosophila have demonstrated that the mutants rutabaga (rut) and dunce (dnc) can show normal adaptation despite their inability to habituate normally (Corfas and Dudai 1990). Within studies investigating C. elegans olfactory plasticity, however, there has been a lack of interest in systematically categorizing these independent processes. For example, Colbert and Bargmann (1995) have demonstrated BZ 'adaptation' by showing that following a 90 min preexposure to BZ (sensed by the AWC neuron), worms exhibit a diminished chemotaxis towards a test spot of this odorant. This decrement in approach was not reversed after washing and centrifugation three times in a buffer, and animals were only able to restore their initial attraction to the odorant after a waiting period of $3 \mathrm{hr}$

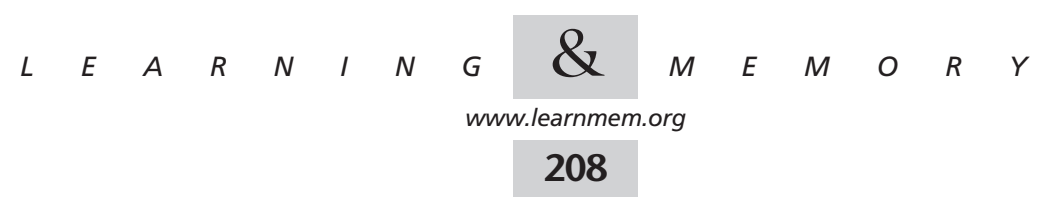


(spontaneous recovery). The animals' ability to restore their olfaction to the odorant attests to the fact that there was no permanent damage done to the worms. However, a similar experiment (W.M. Nuttley, S. Harbinder, and D. van der Kooy, in prep) has demonstrated that dishabituation can be achieved after $\mathrm{BZ}$ preexposure if worms are washed in $\mathrm{dH}_{2} \mathrm{O}$ and given a stronger centrifugal spin. Thus, a phenomenon identified previously as olfactory adaptation has all the critical characteristics of nonassociative learning, suggesting this type of odorant preexposure termed adaptation may be a misclassification and should be considered habituation. Here, we demonstrate that a decrement in DA chemotaxis after preexposure to DA can be caused by both of these processes, and it is the ability of the worms to exhibit a return to naive approach subsequent to a dishabituating stimulus that defined whether adaptation or habituation has occurred. Although habituation has been defined traditionally as a decrement in response after repeated presentation of a single stimulus (Harris 1943; Thompson and Spencer 1966), we consider this equivalent to a continuous presentation of a stimulus for a given period of time, and our controls demonstrate that the criteria for both nonassociative learning and adaptation can be fulfilled by changing only the odorant concentrations (stimulus intensities) and the time of exposure. Changing either the preexposure time or the stimulus intensity may be sufficient to dissociate the processes of adaptation and habituation. Thus, one might predict that a 5 min preexposure to $100 \%$ DA would yield habituation and a $60 \mathrm{~min}$ preexposure to $0.001 \% \mathrm{DA}$ may result in adaptation. This possibility remains to be tested. Alternatively, the longer duration ( $60 \mathrm{~min}$ ) necessary for animals to display a $50 \%$ response decrement after preexposure to $100 \%$ DA (as opposed to the $15 \mathrm{~min}$ needed to see a $25 \%$ response decrement after $0.001 \%$ DA preexposure) may be a reflection of the differences in molecular timing of the two distinct processes. Whereas olfactory habituation may involve readily reversible processes such as receptor phosphorylation or similar manipulations in downstream secondary messenger pathways, the longer time course of adaptation may involve changes in gene transcription leading to down-regulation of olfactory receptors or up-regulation of other unidentified proteins which may act to inhibit chemotaxis via an adaptation-dependent process. Such differences in timing also serve to explain why habituation can be easily reversed with a dishabituating stimulus, whereas adaptation requires a $3 \mathrm{hr}$ spontaneous recovery period before animals can demonstrate a nondecremented response.

Experiments with the odr-10 and odr-1 mutants have allowed genetic dissection of some of the molecular pathways involved in adaptation. Initial baseline approach can be dissociated from DA adaptation, as odr-10 animals exhibit high naive CIs to $100 \%$ DA but do not adapt to this odorant, whereas odr-1 animals have a compromised base- line CI to higher concentrations of DA ( $100 \%$ and $0.1 \%)$ but still demonstrate adaptation. We conclude that the interactions of the high-affinity ODR-10 receptor pathway in AWA with some downstream targets of the proposed low-affinity receptor on AWC (such as the downstream AIZ interneuron) are essential for adaptation and that despite the importance of $o d r-1$ for mediating naive approach to $100 \% \mathrm{DA}$, it is not involved directly in the process of DA adaptation. The ODR-10 receptor alone mediates downstream responses that lead to olfactory habituation and sensitization, however, the current lack of knowledge regarding many specific downstream targets in the odr-10 pathway combined with the fact that $o d r-10$ animals do not approach low DA concentrations presents a challenge to investigations of the role of the $o d r-10$ gene in nonassociative learning.

The low naive approach to high concentrations of DA that was observed in $o d r-1$ mutants is not surprising given the postulated presence of a low-affinity receptor on AWC. The role for an AWC low-affinity receptor in mediating highconcentration DA approach is supported by the observation that $o d r-1$ naive CI to $0.1 \%$ DA was high compared with odr-1 naive approach to the highest DA concentration (100\%). It is possible that this AWC receptor is signaling via an ODR-1 protein and loss of odr-1 leads to loss of the signaling, such that ODR-10 activity alone in AWA can account for the residual approach to DA seen in odr-1 mutants. Although data published previously suggest that approach to $0.1 \%$ DA was not statistically different in odr-1 mutants compared to wild-type worms (Bargmann et al. 1993), we find that the CI to $0.1 \% \mathrm{DA}$ in odr-1 worms is $\sim 20 \%$ lower than wild-type. This deficit, however, was specific to DA because their approach to another AWA-sensed odorant, pyrazine, was the same as wild-type controls. Interestingly, the observation that the maintenance of a compromised baseline approach to DA in odr-1 mutants did not prevent a 50\% response decrement (adaptation) to 100\% DA after $100 \%$ DA preexposure highlights yet another difference between adaptation and habituation because it was noted that habituation-related response decrements are less likely to occur when the baseline approach to low DA concentrations is diminished.

The present findings may allow a distinction among three separate behavioral processes activated by DA preexposure. The first type of behavioral plasticity caused by odorant preexposure is habituation, as characterized by a decrement in DA approach which can dishabituate back to naive levels. The second process underlying a behavioral decrement in response is adaptation, which is likely caused by receptor down-regulation or fatigue because the baseline response can return over time, but can not be dishabituated. The third form of plasticity, which has not been demonstrated before in C. elegans olfaction, is the proposed process of sensitization. We suggest that this form of plasticity may facilitate the behavioral response causing an in-

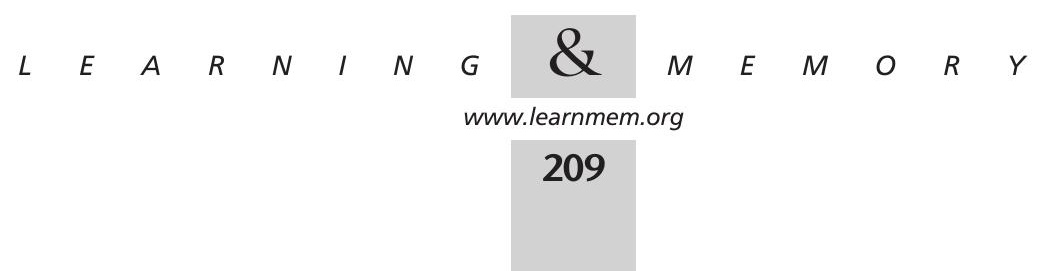


crease above baseline DA approach, or competing with adaptation, may prevent a decrement in DA approach. The development of these paradigms in C. elegans have allowed the exploration of behavioral adaptation at a genetic level. Further analyses at the molecular level will help elucidate the underlying relationship between nonassociative learning and other forms of olfactory plasticity by identifying both common and distinct genetic and cellular pathways.

\section{MATERIALS AND METHODS}

\section{Strains}

The wild-type C. elegans Bristol strain (N2), the odr-10 (ky255) mutant strain (Sengupta et al. 1996) and the odr-1 (n1936) mutant strain (Bargmann et al. 1993) were utilized (mutant strains were provided by the Caenorhabditis elegans Genetic Center). The general culturing techniques used are described by Sulston and Hodgkin (1988). The population of worms was synchronized using the following method (see also Wen at al. 1997): Populations of unsynchronized nondauer plates containing many larval stage 1 and 2 worms were washed off a plate with distilled water $\left(\mathrm{dH}_{2} \mathrm{O}\right)$ into a $15 \mathrm{ml}$ conical centrifuge tube and spun down at $40 \mathrm{~g}$ for $1 \mathrm{~min}$. This procedure causes the older (heavier) worms to form a pellet, leaving the younger worms in the supernatant. The supernatant was then transferred to a second $15 \mathrm{ml}$ conical centrifuge tube and spun down at $150 \mathrm{~g}$ for $1 \mathrm{~min}$. This technique of sequential centrifugation results in a pellet of young larvae that could then be transferred to agar plates seeded with $E$. coli (strain OP50) as a food source. These L1 and L2 larvae from the centrifuged pellet are grown for 3 days resulting in adult worms that are tested at 4 days posthatching, referred to as 3-day-old worms (from the L1/L2 stage). Worms that are younger ( 2 days old) or older ( 4 or 5 days old) in age than the 3-day-old worms used in these experiments were found to exhibit poorer chemotaxis responses to the low concentrations of DA (N. Bernhard and D. van der Kooy, unpubl.).

\section{Materials}

Conditioning and testing were carried out using various different DA concentrations, as specified. Lower concentration DA solutions $(0.01 \%$ and $0.001 \%)$ were prepared the day of the experiment from a stock solution of $1 \% \mathrm{DA}$ (in ethanol) that was made the day prior. High concentration DA and BZ were aliquoted (100\%) or diluted as intermediate concentrations $(0.1 \%, 1 \%, 25 \%)$ on the day of the experiment. All attractants were obtained from Aldrich Chemicals and were always diluted in anhydrous ethyl alcohol. Chemotaxic media (CTX) was prepared using an autoclaved mixture of $1.5 \%$ DIFCO Purified Agar with 0.01 м MOPS buffer ( $\mathrm{pH} 7.2$ with $\mathrm{NH}_{4} \mathrm{OH}$ ). Once the mixture was autoclaved and allowed to cool, $2.5 \mathrm{ml} / 1$ Tween 20 was added and test plates were made by pouring $6 \mathrm{ml}$ of CTX into $100 \mathrm{~mm}$ petri dishes (Fisher Scientific). Agar was distributed evenly throughout the plate and allowed to air dry for 20 min before capping with the lids; plates were generally poured the day prior to the experiment. A plexiglass grid was used under the testing plate to determine the location of placement of the odorant spots and the placement origin of the worms (Fig. 1A). A $1 \mu \mathrm{l}$ spot of $1 \mathrm{~m}$ sodium azide $\left(\mathrm{NaN}_{3}\right)$ was placed on test plates 15 min prior to testing to anesthetize animals as soon as they chose to approach a specific attractant and to prevent the effects of odorant exposure on behavior during the actual test period.

\section{Behavioral Assays}

Worms were washed into $15 \mathrm{ml}$ conical centrifuge tubes from normal growth plates with food using $\mathrm{dH}_{2} \mathrm{O}$ and given a gentle 1 min spin at $40 \mathrm{~g}$. This constituted one wash. All worms were washed twice prior to use. In conformity with the technique used previously by our lab (Wen et al. 1997; Morrisson et al. 1999) a buffer was not used, as there was no noted difference in chemotaxis between worms washed with buffer versus $\mathrm{dH}_{2} \mathrm{O}$ (unpubl.). The resulting pellet of adult worms was transferred carefully to the conditioning CTX plate. Worms were dried gently with a Kimwipe, and DA was pipetted onto agar plugs placed on the lids of the petri dishes. The standard amount of DA used was $5 \mu$ distributed among five agar plugs (variations from this amount are noted in a specific experiment). Control animals were placed on CTX plates with no DA added to the agar plugs. The duration of odorant preexposure ranged between $15 \mathrm{~min}$ for low concentration experiments $(0.001 \%$ and $0.01 \%)$ to 60 and $120 \mathrm{~min}$ for higher concentration experiments $(25 \%, 100 \%)$. The duration of preexposure was varied with different DA concentrations because 15 min was sufficient to induce a response decrement at low DA concentrations, whereas 60 to $120 \mathrm{~min}$ of preexposure were required to see a similar approach decrement at higher DA concentrations. After DA preexposure, $\mathrm{dH}_{2} \mathrm{O}$ was used to wash habituated worms off of conditioning plates and into a $15 \mathrm{ml}$ conical centrifuge tube. Sufficient $\mathrm{dH}_{2} \mathrm{O}$ was added to the tubes to bring the final volume up to $15 \mathrm{ml}$, and the worm suspension was allowed to settle for $5 \mathrm{~min}$. The supernatant was removed and a $5 \mu \mathrm{l}$ spot of worms (containing as many as 200 worms) was aliquoted to the center of the test plate at a point equidistant from the attractant and control spots $(6 \mathrm{~mm}$ wide $\times 20$ $\mathrm{mm}$ long) using an Eppendorf micropipettor with the tip cut short to prevent damage to the animals. The spot of worms was then dried using a Kimwipe, and $1 \mu \mathrm{l}$ spots of DA or control solution (ethanol) were placed on opposite sides of the plate. Plates were parafilmed, and left on the counter for a 60 min test duration. Each plate consisted of 50-200 worms and constitutes an $n=1$.

Dishabituation treatments involved preexposure to DA using the same procedure as above, but instead of allowing to settle for $5 \mathrm{~min}$ in $15 \mathrm{ml}$ of $\mathrm{dH}_{2} \mathrm{O}$, the animals were washed off conditioning plates into a $15 \mathrm{ml}$ conical centrifuge tube with $15 \mathrm{ml}$ of $\mathrm{dH}_{2} \mathrm{O}$. Worms were spun down once at $250 \mathrm{~g}$ for $1 \mathrm{~min}$, the supernatant was removed, and more $\mathrm{dH}_{2} \mathrm{O}$ was added to a final volume of $15 \mathrm{ml}$ prior to centrifuging for a second time at $250 \mathrm{~g}$ for $2 \mathrm{~min}$. The speed of centrifugation varied across the different preexposure experiments, and the different speeds used are noted for those instances. Worms were placed on the test plates in an identical manner as described above for the preexposed only groups. Naive worms were placed on blank CTX plates for the duration of exposure time (i.e., $15 \mathrm{~min}$ for low concentrations, $60 \mathrm{~min}$ for high concentrations), and given the same strong centrifugal spins as the dishabituated groups before testing. There was no difference in chemotaxic approach between unmanipulated naive animals and naive worms exposed only to the dishabituation treatments (data not shown), but as a control, naive groups were always given the dishabituating treatment without DA preexposure. The order of groups tested was randomized within an experiment.

\section{Scoring and Analysis}

After the $60 \mathrm{~min}$ test period, plates were inverted and placed at $4{ }^{\circ} \mathrm{C}$ for $\$ 30 \mathrm{~min}$ prior to counting. Any worms within $20 \mathrm{~mm}$ of the spot were considered to have chosen that odorant (Fig. 1A). A CI

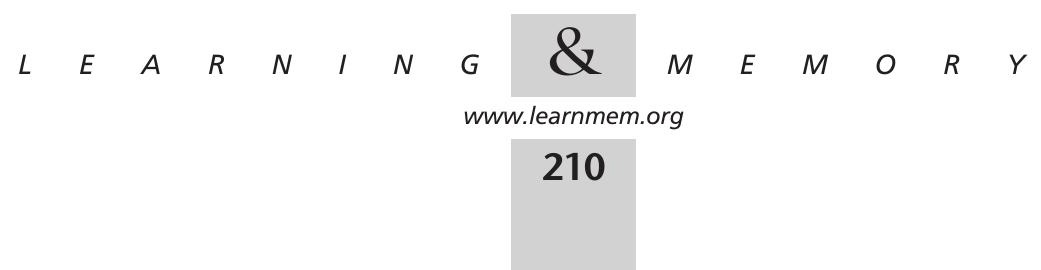


adapted from Bargmann et al. (1993) was calculated based on the numbers of animals at the attractant:

$$
\mathrm{CI}=\frac{\# \text { at test spot }-\# \text { at control spot }}{\text { Total on plate }}
$$

The CI can vary from +1.0 , indicating pure attraction, to -1.0 indicating pure aversion. In general, $<10 \%$ of the worms do not leave the origin, and because this small aggregate of animals in the middle of the plate is likely due to possible damage incurred during the handling procedures, such clumps of worms were not included in the CI calculation. All data were determined to follow a normal distribution using the Kolmogorov-Smirnov test for goodness of fit (Sokal and Rohlf 1995). Mean values, standard deviations, standard error of the mean, regression analysis, t-tests, ANOVAs and posthoc analyses (Neuman-Keuls) were calculated using the Statistica software program (Macintosh StatSoft software version 3.0b).

\section{ACKNOWLEDGMENTS}

This work was supported by the National Science and Engineering Research Council of Canada and N.B. was partially supported by a University of Toronto Open Fellowship. We thank Susan Runciman, Nilo Kaviani, and Rachel Long for their excellent technical support, Nadine Laraya and Inge Scharge for their assistance, Glenn Morrison for his assistance and helpful discussions, and all the members of the van der Kooy lab for their useful suggestions and advice on the manuscript.

The publication costs of this article were defrayed in part by payment of page charges. This article must therefore be hereby marked "advertisement" in accordance with 18 USC section 1734 solely to indicate this fact.

\section{REFERENCES}

Adak, S., Mazumder, A., and Banerjee, R.K. 1996. Probing the active site residues in aromatic donor oxidation in horseradish peroxidase; involvement of an arginine and a tyrosine residue in aromatic donor binding. Biochem. J. 314: 985-991.

Bargmann, C.I. and Horvitz, H.R. 1991. Chemosensory neurons with overlapping functions direct chemotaxis to multiple chemicals in $C$. elegans. Neuron 7: 729-742.

Bargmann, C.I., Thomas, J.H., and Horvitz, H.R. 1990. Chemosensory cell function in the behavior and development of Caenorbabditis elegans. Cold Spring Harbor Symposia on Quantitative Biology LV: 529-538.

Bargmann, C.I., Hartwieg, E., and Horvitz, H.R. 1993. Odorant-selective genes and neurons mediate olfaction in C. elegans. Cell 74: 515-527.

Braun, G. and Bicker, G. 1992. Habituation of an appetitive reflex in the honeybee. J. Neurophysiol. 67: 588-598.

Brown, G.D. 1998. Operational terminology for stimulus exposure (SE) conditioning. Behav. Brain Res. 95: 143-150.

Byrne, J.H. 1987. Cellular analysis of associative learning. Physiol. Rev 67: 329-439.

C. elegans Sequencing Consortium 1998. Genome sequence of the nematode C. elegans: A platform for investigating biology. Science 282: 2012-2018.

Carew, T.J. and Sahley, C.L. 1986. Invertebrate learning and memory: From behavior to molecules. Annu. Rev. Neurosci. 9: 435-487.

Castellucci, V., Pinsker, H., Kupferman I., and Kandel, E.R. 1970. Neuronal mechanisms of habituation and dishabituation if the gill-withdrawal reflex in Aplysia. Science 167: 1745-1748.

Chen, T.Y. and Yau, K.W. 1994. Direct modulation by $\mathrm{Ca}^{2+}$-calmodulin of cyclic nucleotide-activated channel of rat olfactory receptor neurons. Nature 368: 545-548.

Chou, J.H., Troemel, E.R., Sengupta, P., Colbert, L., Tong, L., Tobin, D.M., Roayaie, K., Crump, J.G., Dwyer, N.D., and Bargmann, C.I. 1996. Olfactory recognition and discrimination in Caenorbabditis elegans. Cold Spring Harbor Symposia on Quantitative Biology LXI: 157-164.
Chalfie, M. and Sulston, J. 1981. Developmental genetics of the mechanosensory neurons of C. elegans. Dev. Biol. 82: 358-370.

Coburn, C. and Bargmann, C.I. 1996. A putative cyclic nucleotide-gated channel is required for sensory development and function in $C$. elegans. Neuron 17: 695-706.

Cohen, T.E., Kaplan, S.W., Kandel, E.R. and Hawkins, R.D. 1997. A simplified preparation for relating cellular events to behavior: Mechanisms contributing to habituation, dishabituation, and sensitization of the Aplysia gill-withdrawal reflex. J. Neurosci. 17: 2886-2899.

Colbert, H.A. and Bargmann, C.I. 1995. Odorant-specific adaptation pathways generate olfactory plasticity in C. elegans. Neuron 14: 803-812.

- 1997. Environmental signals modulate olfactory acuity, discrimination, and memory in Caenorhabditis elegans. Learn. Mem. 4: 179-191.

Colbert, H.A., Smith, T.L., and Bargmann, C.I. 1997. osm-9, a novel protein with structural similarity to channels, is required for olfaction, mechanosensation and olfactory adaptation in Caenorhabditis elegans. J. Neurosci. 17: 8259-8269.

Corfas, G. and Dudai, Y. 1988. Habituation and dishabituation of a cleaning reflex in normal and mutant Drosophila. J. Neurosci. 9: 56-62.

-1990. Adaptation and fatigue of a mechanosensory neuron in wild-type Drosophila and in memory mutants. J. Neurosci. 10: 491-499.

Croll, N. 1975. Indoleakylamines in the co-ordination of nematode behavioral activities. Can. J. Zool. 53: 894-903.

Dawson, T., Arriza, J., Jaworski, D., Borisy, F., Attramadal, H., Lefkowitz, R., and Ronnet, G. 1993. $\beta$-adrenergic receptor kinase-2 and $\beta$-arrestin-2 as mediators of odorant-induced desensitization. Science 259: 825-829

Dizhoor, A.M., Ray, S., Kumar, S., Niemi, G., Spencer, M., Brolley, D., Walsh, K.A., Philpov, P.P., Hurley, J.B., and Stryer, L. 1991. Recoverin: A calcium sensitive activator of retinal rod guanylate cyclase. Science 251: 915-918.

Eriksson, O., Fontain, E., and Bernardi, P. 1998. Chemical modification of arginines by 2,3-butanedione and phenylglyoxal causes closure of the mitachondrial permeability transition pore. J. Biol. Chem. 273: 12669-12674.

Gannon, T.N. and C.H. Rankin. 1995. Methods of studying behavioral plasticity in Caenorhabditis elegans. In Caenorhabditis elegans: Modern biological analysis of an organism. (ed. H.F. Epstein and D.C. Shakes), pp. 205-223. Academic Press, San Diego, California.

Groves, P.M. and Thompson, R.F. 1970. Habituation: A dual process theory. Psych. Rev. 77: 419-450.

Hall, D.H. and Russel, L.R. 1991. The posterior nervous system of the nematode Caenorhabditis elegans: Serial reconstruction of identified neurons and complete pattern of synaptic interactions. J. Neurosci. 11: 1-22.

Hammer, M. 1997. The neural basis of associative reward learning in honeybees. Trends Neurosci. 20: 245-252.

Harris, J.D. 1943. Habituatory response decrement in the intact organism. Psych. Bul. 40: 385-422.

Hedgecock, E.M. and Russell, R.L. 1975. Normal and mutant thermotaxis in the nematode, Caenorhabditis elegans. Proc. Natl. Acad. Sci. 72: 4061-4065.

Hellerstern, F., Malaka, R., and Hammer, M. 1998. Backward inhibitory learning in honeybees: A behavioral analysis of reinforcement processing. Learn. Mem. 4: 429-444.

Kawamura, S. and Murakami, M. 1991. Calcium-dependent regulation of cyclic GMP phosphodiesterase by a protein from frog retinal rods. Nature 349: 420-423.

Kramer, R.H. and Siegelbaum, S.A. 1992. Intracellular $\mathrm{Ca}^{2}+$ regulates the sensitivity of cyclic nucleotide gated channels in olfactory receptor neurons. Neuron 9: 897-906.

Kurahashi, T. and Menini A. 1997. Mechanism of odorant adaptation in the olfactory receptor cell. Nature 385: 725-729.

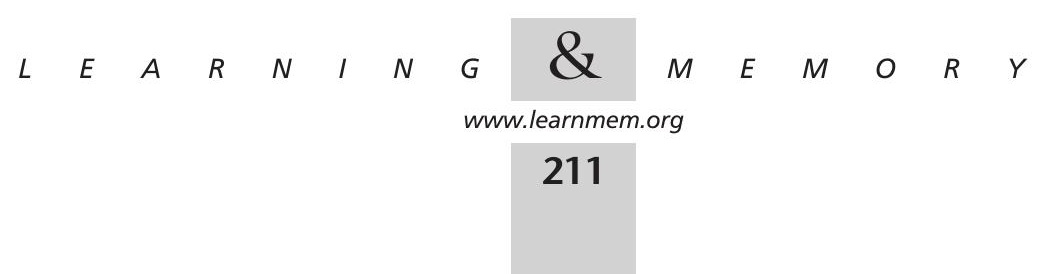


Leinders-Zufall, T., Greer, C.A., Shepherd, G.M., and Zufall, F. 1998. Visualizing odor detection in olfactory cilia by calcium imaging. Ann. NY Acad. Sci. 855: 205-207.

Marcus, E.A., Nolen, T.G., Rankin, C.H., and Carew, T.J. 1988. Behavioral dissociation of dishabituation, sensitization and inhibition in Aplysia. Science 241: 210-213

Morrison, G.E., Wen, J.Y.M., Runciman, S., and van der Kooy, D. 1999 Olfactory associative learning in Caenorhabditis elegans is impaired in lrn-1 and lrn-2 mutants. Behav. Neurosci. 113: 358-367.

Ochoa, E.L., Li, L., and McNamee, M.G. 1990. Desensitization of central cholinergic mechanisms and neuroadaptation to nicotine. Mol. Neurobiol. 4: 251-287.

Quinn, W.G., Harris, W.A., and Benzer, S. 1974. Conditioned behavior in Drosophila melanogaster. Proc. Natl. Acad. Sci. 71: 708-712.

Rankin, C.H., Beck, C.D.O., and Chiba, C.M. 1990. Caenorhabditis elegans: A new model system for the study of learning and memory. Behav. Brain Res. 37: 89-92.

Rauschecker, K. and Korte, M. 1993. Auditory compensation for early blindness in cat cerebral cortex. J. Neurosci. 13: 4538-4548.

Roayaie, K., Crump, J.G., Sagasti, A., and Bargmann, C.I. 1998. The G $\alpha$ protein ODR-3 mediates olfactory and nociceptive function and controls cilium morphogenesis in C. elegans olfactory neurons. Neuron 20: 55-67.

Rogers, R.F. and Matzel, L.D. 1995. Higher-order associative processing in Hermissenda suggests multiple sites of neuronal modulation. Learn. Mem. 2: 279-298.

Sengupta, P., Chou, J.H., and Bargmann, C.I. 1996. odr-10 encodes a seven transmembrane domain olfactory receptor required for responses to the odorant diacetyl. Cell 84: 899-909.

Sokal, R.R. and Rohlf, F.J. 1995. Biometry: The principles and practice of statistics in biological research, pp. 708-715. W.H. Freeman and Company, New York, NY.

Sulston, J. and Hodgkin, J. 1988. Methods. In The nematode Caenorhabditis elegans (ed. W.B. Woods), pp. 587-606. Cold Spring Harbor Laboratory Press, Cold Spring Harbor, NY.

Sulston, J., Du, Z., Thomas, K., Wilson, R., Hillier, L., Staden, R., Halloran, N., Green, P., Thierry-Mieg, J., Qiu, L., et al. 1992. The C. elegans genome sequencing project: A beginning. Nature 356: 37-41.
Thompson, R.F. and Spencer, W.A. 1966. Habituation: A model phenomenon for the study of neuronal substrates of behavior. Psych. Rev. 173: 16-43.

Troemel, E.R., Kimmel, B.E., and Bargmann, C.I. 1997. Reprogramming chemotaxis responses: Sensory neurons define olfactory preferences in C. elegans. Cell 91: 161-169.

Tully, T. and Quinn, W.G. 1985. Classical conditioning and retention in normal and mutant Drosophila melanogaster. J. Comp. Physiol. 157: 263-277.

Walter, E.T., Carew, T.J., and Kandel, E.R. 1979. Classical conditioning in Aplysia Californica. Proc. Natl. Acad. Sci. 76: 6675-6679.

- 1981. Associative learning in Aplysia: Evidence for conditioned fear in invertebrates. Science 211: 504-506.

Ward, S. 1973. Chemotaxis by the nematode Caenorbabditis elegans: Identification of attractants and analysis of the response by mutants Proc. Natl. Acad. Sci. 70: 817-821.

Wen, J., Kumar, N., Morrison, G.E., Runciman, S., Rambaldini, G., Rousseau, J., and van der Kooy, D. 1997. Mutations that prevent associative learning in C. elegans. Behav. Neurosci. 111: 342-353

White, J.G., Southgate, E., Thompson, J.N., and Brenner, S. 1986. The structure of the nervous system in the nematode Caenorbabditis elegans. Phil. Trans. R. Soc. Lond. B. Biol. Sci. 314: 1-340.

Wicks, S.R. and Rankin, C.H. 1996. The integration of antagonistic reflexes revealed by laser ablation of identified neurons determines habituation kinetics of the Caenorhabditis elegans tap withdrawal response. $J$. Comp. Physiol. 176: 675-685.

- 1997. Effects of tap withdrawal esponse habituation on other withdrawal behaviors: The localization of habituation in the nematode Caenorhabditis elegans. Behav. Neurosci. 11: 354-368.

Zhang, Y., Chou, J.H., Bradley, J., Bargmann, C.I., and Zinn, K. 1997. The Caenorhabditis elegans seven-transmembrane protein ODR-10 functions as an odorant receptor in mammalian cells. Proc. Natl. Acad. Sci. 94: 12162-12167.

Received August 20, 1999; accepted in revised form June 26, 2000. 


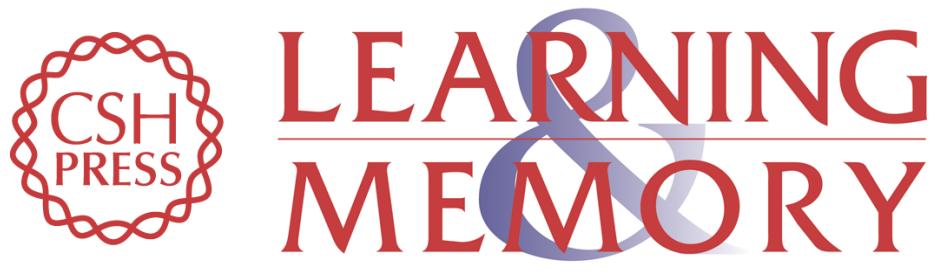

\section{A Behavioral and Genetic Dissection of Two Forms of Olfactory Plasticity in Caenorhabditis elegans: Adaptation and Habituation}

Nirit Bernhard and Derek van der Kooy

Learn. Mem. 2000, 7:

Access the most recent version at doi:10.1101//m.7.4.199

References This article cites 52 articles, 21 of which can be accessed free at: http://learnmem.cshlp.org/content/7/4/199.full.html\#ref-list-1

License

Email Alerting Receive free email alerts when new articles cite this article - sign up in the box at the Service top right corner of the article or click here. 